\title{
Lucid Data Dreaming for Video Object Segmentation
}

\author{
Anna Khoreva ${ }^{1}$ (I) Rodrigo Benenson ${ }^{2} \cdot$ Eddy $\mathrm{Ig}^{3} \cdot$ Thomas Brox $^{3} \cdot$ Bernt Schiele $^{1}$
}

Received: 11 June 2018 / Accepted: 5 February 2019 / Published online: 15 March 2019

(c) The Author(s) 2019

\begin{abstract}
Convolutional networks reach top quality in pixel-level video object segmentation but require a large amount of training data $(1 \mathrm{k}-100 \mathrm{k})$ to deliver such results. We propose a new training strategy which achieves state-of-the-art results across three evaluation datasets while using $20 \times-1000 \times$ less annotated data than competing methods. Our approach is suitable for both single and multiple object segmentation. Instead of using large training sets hoping to generalize across domains, we generate in-domain training data using the provided annotation on the first frame of each video to synthesize- "lucid dream" (in a lucid dream the sleeper is aware that he or she is dreaming and is sometimes able to control the course of the dream)—plausible future video frames. In-domain per-video training data allows us to train high quality appearance- and motion-based models, as well as tune the post-processing stage. This approach allows to reach competitive results even when training from only a single annotated frame, without ImageNet pre-training. Our results indicate that using a larger training set is not automatically better, and that for the video object segmentation task a smaller training set that is closer to the target domain is more effective. This changes the mindset regarding how many training samples and general "objectness" knowledge are required for the video object segmentation task.
\end{abstract}

Keywords Video object segmentation $\cdot$ Synthetic data $\cdot$ Data augmentation $\cdot$ Convolutional neural networks

\section{Introduction}

In the last years the field of localizing objects in videos has transitioned from bounding box tracking (Kristan et al. 2015, 2014, 2016) to pixel-level segmentation (Li et al. 2013; Prest et al. 2012; Perazzi et al. 2016; Vojir and Matas 2017). Given a first frame labelled with the foreground object masks, one

Communicated by Xiaoou Tang.

Anna Khoreva

khoreva@mpi-inf.mpg.de

Rodrigo Benenson

benenson@google.com

Eddy Ilg

ilg@cs.uni-freiburg.com

Thomas Brox

brox@cs.uni-freiburg.com

Bernt Schiele

schiele@mpi-inf.mpg.de

1 Max Planck Institute for Informatics, Saarbrücken, Germany

2 Google, Menlo Park, USA

3 University of Freiburg, Freiburg, Germany aims to find the corresponding object pixels in future frames. Segmenting objects at the pixel level enables a finer understanding of videos and is helpful for tasks such as video editing, rotoscoping, and summarisation.

Top performing results are currently obtained using convolutional networks (convnets) (Jampani et al. 2016; Caelles et al. 2017; Khoreva et al. 2016; Bertinetto et al. 2016; Held et al. 2016; Nam et al. 2016b). Like most deep learning techniques, convnets for video object segmentation benefit from large amounts of training data. Current state-of-the-art methods rely, for instance, on pixel accurate foreground/background annotations of $\sim 2 \mathrm{k}$ video frames (Jampani et al. 2016; Caelles et al. 2017), 10k images (Khoreva et al. 2016), or even more than 100k annotated samples for training (Voigtlaender and Leibe 2017b). Labelling images and videos at the pixel level is a laborious task (compared e.g. to drawing bounding boxes for detection), and creating a large training set requires significant annotation effort.

In this work we aim to reduce the necessity for such large volumes of training data. It is traditionally assumed that convnets require large training sets to perform best. We show that for video object segmentation having a larger training set is not automatically better and that improved results can 


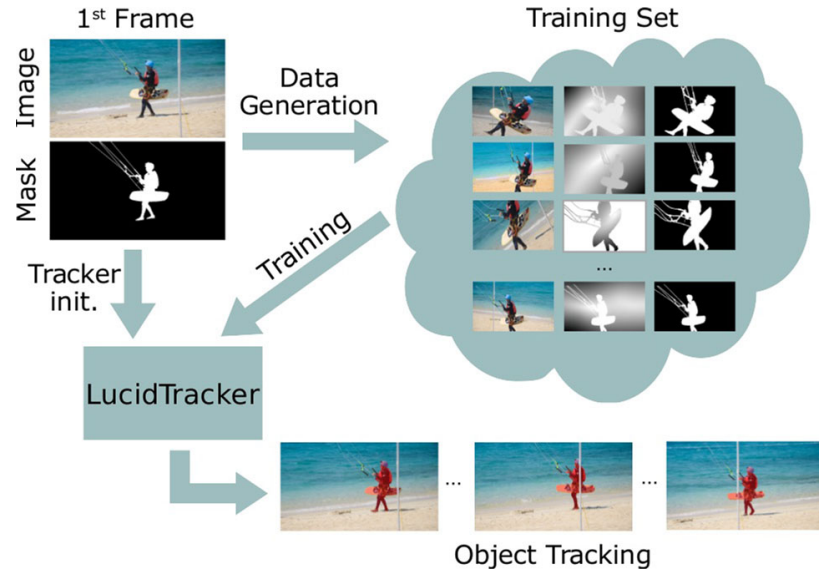

Fig. 1 Starting from scarce annotations we synthesize in-domain data to train a specialized pixel-level video object segmenter for each dataset or even each video sequence

be obtained by using $20 \times-1000 \times$ less training data than previous approaches (Caelles et al. 2017; Khoreva et al. 2016; Voigtlaender and Leibe 2017b). The main insight of our work is that for video object segmentation using few training frames (1-100) in the target domain is more useful than using large training volumes across domains $(1 \mathrm{k}-100 \mathrm{k})$.

To ensure a sufficient amount of training data close to the target domain, we develop a new technique for synthesizing training data particularly tailored for the pixel-level video object segmentation scenario. We call this data generation strategy "lucid dreaming", where the first frame and its annotation mask are used to generate plausible future frames of the videos (see Fig. 1). The goal is to produce a large training set of reasonably realistic images which capture the expected appearance variations in future video frames, and thus is, by design, close to the target domain.

Our approach is suitable for both single and multiple object segmentation in videos. Enabled by the proposed data generation strategy and the efficient use of optical flow, we are able to achieve high quality results while using only $\sim 100$ individual annotated training frames. Moreover, in the extreme case with only a single annotated frame and zero pre-training (i.e. without ImageNet pre-training), we still obtain competitive video object segmentation results.

In summary, our contributions are the following:

1. We propose "lucid data dreaming", an automated approach to synthesize training data for the convnetbased pixel-level video object segmentation that leads to top results for both single and multiple object segmentation. $^{1}$

2. We conduct an extensive analysis to explore the factors contributing to our good results.

\footnotetext{
${ }^{1}$ Lucid data dreaming synthesis implementation is available at https:// www.mpi-inf.mpg.de/lucid-data-dreaming.
}

3. We show that training a convnet for video object segmentation can be done with only few annotated frames. We hope these results will affect the trend towards even larger training sets, and popularize the design of video segmentation convnets with lighter training needs.

With the results for multiple object segmentation we took the second place in the 2017 DAVIS Challenge on Video Object Segmentation (Pont-Tuset et al. 2017b). A summary of the proposed approach was provided online (Khoreva et al. 2017). This paper significantly extends (Khoreva et al. 2017) with in-depth discussions on the method, more details of the formulation, its implementation, and its variants for single and multiple object segmentation in videos. It also offers a detailed ablation study and an error analysis as well as explores the impact of varying number of annotated training samples on the video segmentation quality.

\section{Related Work}

Box Tracking Classic work on video object tracking focused on bounding box tracking. Many of the insights from these works have been re-used for video object segmentation. Traditional box tracking smoothly updates across time a linear model over hand-crafted features (Henriques et al. 2012; Breitenstein et al. 2009; Kristan et al. 2014). Since then, convnets have been used as improved features (Danelljan et al. 2015; Ma et al. 2015; Wang et al. 2015), and eventually to drive the tracking itself (Held et al. 2016; Bertinetto et al. 2016; Tao et al. 2016; Nam et al. 2016a, b). Contrary to traditional box trackers (e.g. Henriques et al. 2012), convnetbased approaches need additional data for pre-training and learning the task.

Video Object Segmentation In this paper we focus on generating a foreground versus background pixel-wise object labelling for each video frame starting from a first manually annotated frame. Multiple strategies have been proposed to solve this task.

Box-to-Segment First a box-level track is built, and a space-time grabcut-like approach is used to generate per frame segments (Xiao and Lee 2016).

Video Saliency This group of methods extracts the main foreground object pixel-level space-time tube. Both handcrafted models (Faktor and Irani 2014; Papazoglou and Ferrari 2013) or trained convnets (Tokmakov et al. 2017; Jain et al. 2017; Song et al. 2018) have been considered. Because these methods ignore the first frame annotation, they fail in videos where multiple salient objects move (e.g. flock of penguins).

Space-Time Proposals These methods partition the spacetime volume, and then the tube overlapping most with the 
first frame mask annotation is selected as tracking output (Grundmann et al. 2010; Perazzi et al. 2015; Chang et al. 2013).

Mask Propagation Appearance similarity and motion smoothness across time is used to propagate the first frame annotation across the video (Maerki et al. 2016; Wang and Shen 2017; Tsai et al. 2016). These methods usually leverage optical flow and long term trajectories.

Convnets Following the trend in box tracking, recently convnets have been proposed for video object segmentation. Caelles et al. (2017) trains a generic object saliency network, and fine-tunes it per-video (using the first frame annotation) to make the output sensitive to the specific object of interest. Khoreva et al. (2016) uses a similar strategy, but also feeds the mask from the previous frame as guidance for the saliency network. Voigtlaender and Leibe (2017b) incorporates online adaptation of the network using the predictions from previous frames. Chandra et al. (2018) extends the Gaussian-CRF approach to videos by exploiting spatio-temporal connections for pairwise terms and relying on unary terms from (Voigtlaender and Leibe 2017b). Finally Jampani et al. (2016) mixes convnets with ideas of bilateral filtering. Our approach also builds upon convnets.

What makes convnets particularly suitable for the task, is that they can learn what are the common statistics of appearance and motion patterns of objects, as well as what makes them distinctive from the background, and exploit this knowledge when segmenting a particular object. This aspect gives convnets an edge over traditional techniques based on low-level hand-crafted features.

Our network architecture is similar to Caelles et al. (2017) and Khoreva et al. (2016). Other than implementation details, there are three differentiating factors. One, we use a different strategy for training: (Caelles et al. 2017; Jampani et al. 2016; Chandra et al. 2018; Voigtlaender and Leibe 2017b) rely on consecutive video training frames and (Khoreva et al. 2016) uses an external saliency dataset, while our approach focuses on using the first frame annotations provided with each targeted video benchmark without relying on external annotations. Two, our approach exploits optical flow better than these previous methods. Three, we describe an extension to seamlessly handle segmentation of multiple objects.

Interactive Video Segmentation Interactive segmentation (Nagaraja et al. 2015; Jain and Grauman 2016; Spina and Falcão 2016; Wang et al. 2014) considers more diverse user inputs (e.g. strokes), and requires interactive processing speed rather than providing maximal quality. Albeit our technique can be adapted for varied inputs, we focus on maximizing quality for the non-interactive case with no-additional hints along the video.

Semantic Labelling Like other convnets in this space (Jampani et al. 2016; Caelles et al. 2017; Khoreva et al. 2016), our architecture builds upon the insights from the semantic labelling networks (Zhao et al. 2017; Lin et al. 2016; Wu et al. 2016; Bansal et al. 2017). Because of this, the flurry of recent developments should directly translate into better video object segmentation results. For the sake of comparison with previous work, we build upon the well established VGG DeepLab architecture (Chen et al. 2016).

Synthetic Data Like our approach, previous works have also explored synthesizing training data. Synthetic renderings (Mayer et al. 2016), video game environment (Richter et al. 2016), mix-synthetic and real images (Varol et al. 2017; Chen et al. 2016; Dosovitskiy et al. 2015) have shown promise, but require task-appropriate $3 \mathrm{~d}$ models. Compositing real world images provides more realistic results, and has shown promise for object detection (Georgakis et al. 2017; Tang et al. 2013), text localization (Gupta et al. 2016) and pose estimation (Pishchulin et al. 2012).

The closest work to ours is Park and Ramanan (2015), which also generates video-specific training data using the first frame annotations. They use human skeleton annotations to improve pose estimation, while we employ pixel-level mask annotations to improve video object segmentation.

\section{LucidTracker}

Section 3.1 describes the network architecture used, and how RGB and optical flow information are fused to predict the next frame segmentation mask. Section 3.2 discusses different training modalities employed with the proposed video object segmentation system. In Sect. 4 we discuss the training data generation, and Sects. 5/6 report results for single/multiple object segmentation in videos.

\subsection{Architecture}

Approach We model video object segmentation as a mask refinement task (mask: binary foreground/ background labelling of the image) based on appearance and motion cues. From frame $t-1$ to frame $t$ the estimated mask $M_{t-1}$ is propagated to frame $t$, and the new mask $M_{t}$ is computed as a function of the previous mask, the new image $\mathcal{I}_{t}$, and the optical flow $\mathcal{F}_{t}$, i.e. $M_{t}=f\left(\mathcal{I}_{t}, \mathcal{F}_{t}, M_{t-1}\right)$. Since objects have a tendency to move smoothly through space in time, there are little changes from frame to frame and mask $M_{t-1}$ can be seen as a rough estimate of $M_{t}$. Thus we require our trained convnet to learn to refine rough masks into accurate masks. Fusing the complementary image $\mathcal{I}_{t}$ and motion flow $\mathcal{F}_{t}$ enables to exploits the information inherent to video and enables the model to segment well both static and moving objects. 


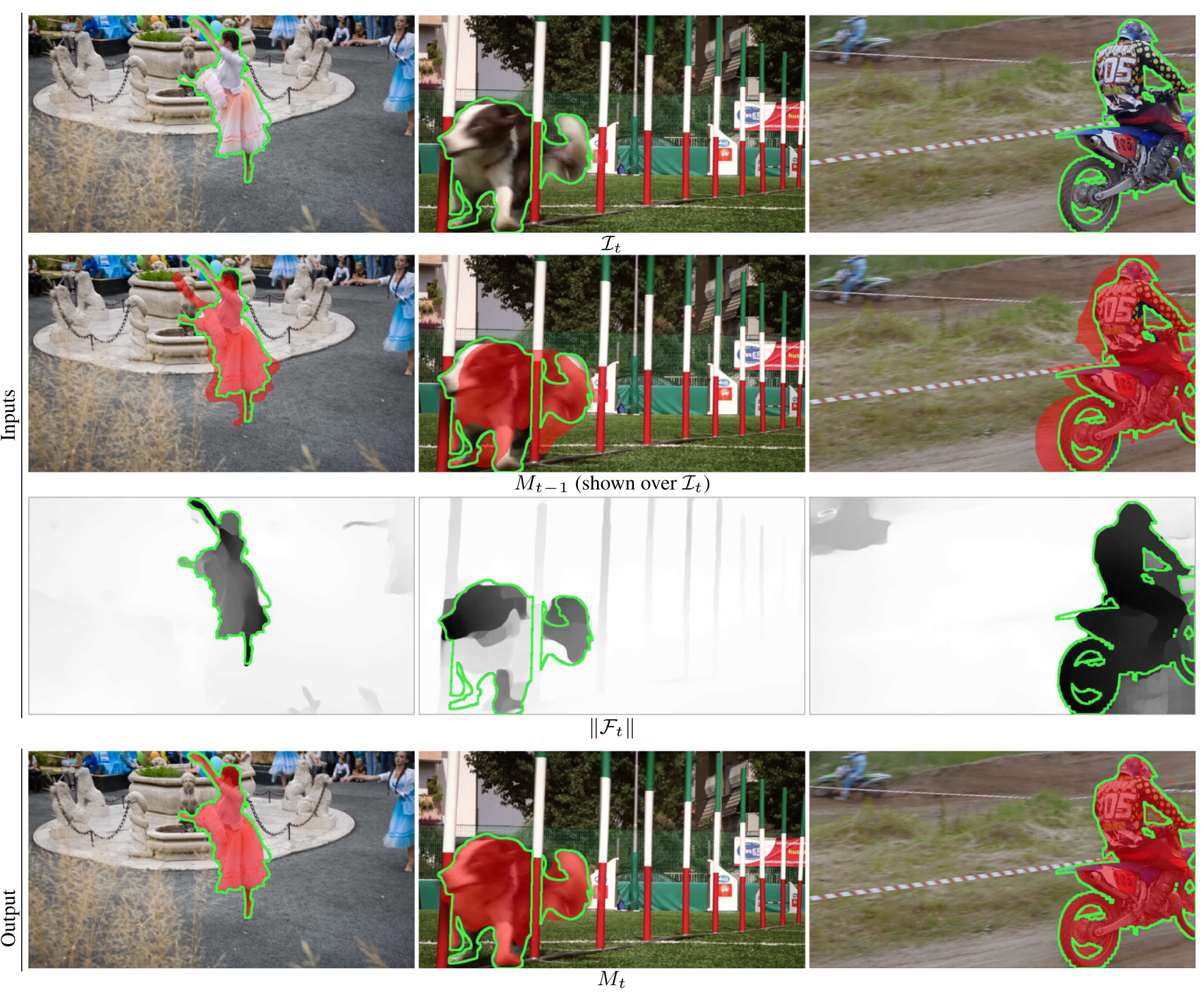

Fig. 2 Data flow examples. $\mathcal{I}_{t},\left\|\mathcal{F}_{t}\right\|, M_{t-1}$ are the inputs, $M_{t}$ is the resulting output. Green boundaries outline the ground truth segments. Red overlay indicates $M_{t-1}, M_{t}$

Note that this approach is incremental, does a single forward pass over the video, and keeps no explicit model of the object appearance at frame $t$. In some experiments we adapt the model $f$ per video, using the annotated first frame $\mathcal{I}_{0}, M_{0}$. However, in contrast to traditional techniques (Henriques et al. 2012), this model is not updated while we process the video frames, thus the only state evolving along the video is the mask $M_{t-1}$ itself.

First Frame In the video object segmentation task of our interest the mask for the first frame $M_{0}$ is given. This is the standard protocol of the benchmarks considered in Sects. 5 and 6. If only a bounding box is available on the first frame, then the mask could be estimated using grabcut-like techniques (Rother et al. 2004; Tang et al. 2016).

RGB Image $\mathcal{I}$ Typically a semantic labeller generates pixelwise labels based on the input image (e.g. $M=g(\mathcal{I})$ ). We use an augmented semantic labeller with an input layer modified to accept 4 channels (RGB + previous mask) so as to generate outputs based on the previous mask estimate, e.g. $M_{t}=f_{\mathcal{I}}\left(\mathcal{I}_{t}, M_{t-1}\right)$. Our approach is general and can leverage any existing semantic labelling architecture. We select the DeepLabv2 architecture with VGG base network (Chen et al. 2016), which is comparable to (Jampani et al. 2016; Caelles et al. 2017; Khoreva et al. 2016); FusionSeg (Jain et al. 2017) uses ResNet.

Optical Flow $\mathcal{F}$ We use flow in two complementary ways. First, to obtain a better initial estimate of $M_{t}$ we warp $M_{t-1}$ using the flow $\mathcal{F}_{t}: M_{t}=f_{\mathcal{I}}\left(\mathcal{I}_{t}, w\left(M_{t-1}, \mathcal{F}_{t}\right)\right.$ ); we call this "mask warping". Second, we use flow as a direct source of information about the mask $M_{t}$. As can be seen in Fig. 2, when the object is moving relative to background, the flow magnitude $\left\|\mathcal{F}_{t}\right\|$ provides a very rea- 


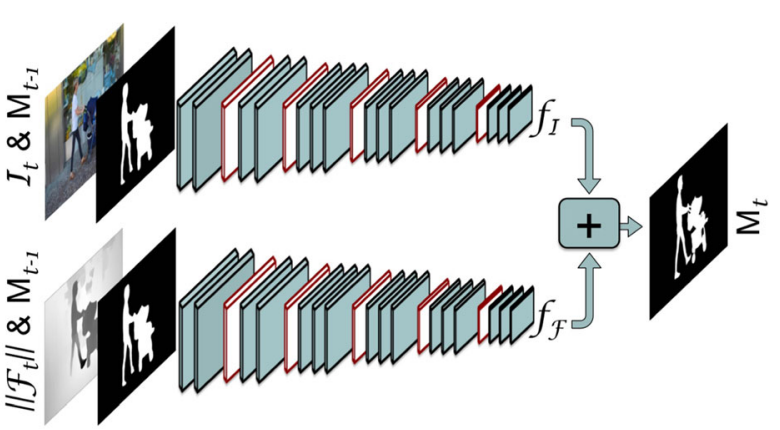

(a) Two streams architecture, where image $\mathcal{I}_{t}$ and optical flow information $\left\|\mathcal{F}_{t}\right\|$ are used to update mask $M_{t-1}$ into $M_{t}$. See equation 1

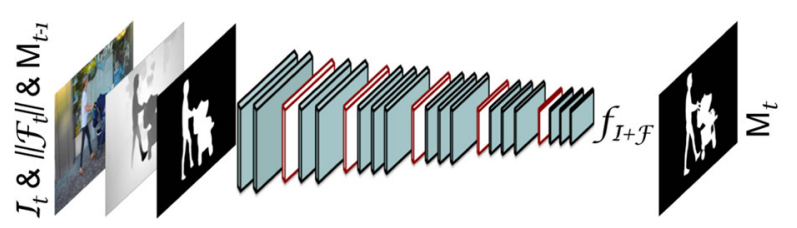

(b) One stream architecture, where 5 input channels: image $\mathcal{I}_{t}$, optical flow information $\left\|\mathcal{F}_{t}\right\|$ and mask $M_{t-1}$ are used to estimate mask $M_{t}$

Fig. 3 Overview of the proposed one and two streams architectures. See Sect. 3.1

sonable estimate of the mask $M_{t}$. We thus consider using a convnet specifically for mask estimation from flow: $M_{t}=$ $f_{\mathcal{F}}\left(\left\|\mathcal{F}_{t}\right\|, w\left(M_{t-1}, \mathcal{F}_{t}\right)\right)$, and merge it with the image-only version by naive averaging

$M_{t}=0.5 \cdot f_{\mathcal{I}}\left(\mathcal{I}_{t}, \ldots\right)+0.5 \cdot f_{\mathcal{F}}\left(\left\|\mathcal{F}_{t}\right\|, \ldots\right)$.

We use the state-of-the-art optical flow estimation method FlowNet2.0 (Ilg et al. 2017), which itself is a convnet that computes $\mathcal{F}_{t}=h\left(\mathcal{I}_{t-1}, \mathcal{I}_{t}\right)$ and is trained on synthetic renderings of flying objects (Mayer et al. 2016). For the optical flow magnitude computation we subtract the median motion for each frame, average the magnitude of the forward and backward flow and scale the values per-frame to [0;255], bringing it to the same range as RGB channels.

The loss function is the sum of cross-entropy terms over each pixel in the output map (all pixels are equally weighted). In our experiments $f_{\mathcal{I}}$ and $f_{\mathcal{F}}$ are trained independently, via some of the modalities listed in Sect. 3.2. Our two streams architecture is illustrated in Fig. 3a.

We also explored expanding our network to accept 5 input channels (RGB + previous mask + flow magnitude) in one stream: $M_{t}=f_{\mathcal{I}+\mathcal{F}}\left(\mathcal{I}_{t},\left\|\mathcal{F}_{t}\right\|, w\left(M_{t-1}, \mathcal{F}_{t}\right)\right)$, but did not observe much difference in the performance compared to naive averaging, see experiments in Sect. 5.4.3. Our one stream architecture is illustrated in Fig. 3b. One stream network is more affordable to train and allows to easily add extra input channels, e.g. providing additionally semantic information about objects.

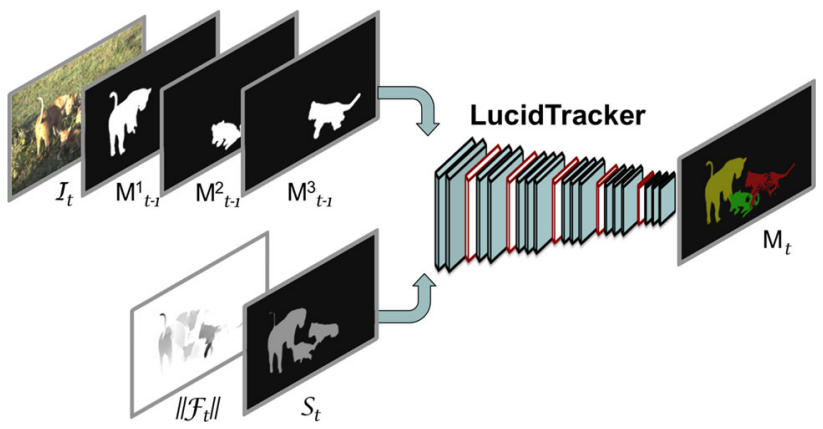

Fig. 4 Extension of LucidTracker to multiple objects. The previous frame mask for each object is provided in a separate channel. We additionally explore using optical flow $\mathcal{F}$ and semantic segmentation $\mathcal{S}$ as additional inputs. See Sect. 3.1

Multiple Objects The proposed framework can easily be extended to segmenting multiple objects simultaneously. Instead of having one additional input channel for the previous frame mask we provide the mask for each object instance in a separate channel, expanding the network to accept $3+N$ input channels (RGB $+N$ object masks): $M_{t}=f_{\mathcal{I}}\left(\mathcal{I}_{t}, w\left(M_{t-1}^{1}, \mathcal{F}_{t}\right), \ldots, w\left(M_{t-1}^{N}, \mathcal{F}_{t}\right)\right)$, where $N$ is the number of objects annotated on the first frame.

For multiple object segmentation we employ a onestream architecture for the experiments, using optical flow $\mathcal{F}$ and semantic segmentation $\mathcal{S}$ as additional input channels: $M_{t}=f_{\mathcal{I}+\mathcal{F}+\mathcal{S}}\left(\mathcal{I}_{t},\left\|\mathcal{F}_{t}\right\|, \mathcal{S}_{t}, w\left(M_{t-1}^{1}, \mathcal{F}_{t}\right), \ldots, w\right.$ $\left.\left(M_{t-1}^{N}, \mathcal{F}_{t}\right)\right)$. This allows to leverage the appearance model with semantic priors and motion information. See Fig. 4 for an illustration.

The one-stream network is trained with multi-class cross entropy loss and is able to segment multiple objects simultaneously, sharing the feature computation for different instances. This allows to avoid a linear increase of the cost with the number of objects. In our preliminary results using a single architecture also provides better results than segmenting multiple objects separately, one at a time; and avoids the need to design a merging strategy amongst overlapping tracks.

Semantic Labels $\mathcal{S}$ To compute the pixel-level semantic labelling $\mathcal{S}_{t}=h\left(\mathcal{I}_{t}\right)$ we use the state-of-the-art convnet PSPNet (Zhao et al. 2017), trained on Pascal VOC12 (Everingham et al. 2015). Pascal VOC12 annotates 20 categories, yet we want to track any type of objects. $\mathcal{S}_{t}$ can also provide information about unknown category instances by describing them as a spatial mixture of known ones (e.g. a sea lion might looks like a dog torso, and the head of cat). As long as the predictions are consistent through time, $\mathcal{S}_{t}$ will provide a useful cue for segmentation. Note that we only use $\mathcal{S}_{t}$ for the multi-object segmentation challenge, discussed in Sect. 6. In the same way as for the optical flow we scale $\mathcal{S}_{t}$ to bring all the channels to the same range. 
We additionally experiment with ensembles of different variants, that allows to make the system more robust to the challenges inherent in videos. For our main results on the multiple object segmentation task we consider an ensemble of four models: $M_{t}=0.25 \cdot\left(f_{\mathcal{I}+\mathcal{F}+\mathcal{S}}+f_{\mathcal{I}+\mathcal{F}}+f_{\mathcal{I}+\mathcal{S}}\right.$ $+f_{\mathcal{I}}$ ), where we merge the outputs of the models by naive averaging. See Sect. 6 for more details.

Temporal Coherency To improve the temporal coherency of the proposed video object segmentation framework we introduce an additional step into the system. Before providing as input the previous frame mask warped with the optical flow $w\left(M_{t-1}, \mathcal{F}_{t}\right)$, we look at frame $t-2$ to remove inconsistencies between the predicted masks $M_{t-1}$ and $M_{t-2}$. In particular, we split the mask $M_{t-1}$ into connected components and remove all components from $M_{t-1}$ which do not overlap with $M_{t-2}$. This way we remove possibly spurious blobs generated by our model in $M_{t-1}$. Afterwards we warp the "pruned" mask $\widetilde{M}_{t-1}$ with the optical flow and use $w\left(\widetilde{M}_{t-1}, \mathcal{F}_{t}\right)$ as an input to the network. This step is applied only during inference, it mitigates error propagation issues, as well as help generating more temporally coherent results.

Post-processing As a final stage of our pipeline, we refine per-frame $t$ the generated mask $M_{t}$ using DenseCRF (Krähenbühl and Koltun 2011). This adjusts small image details that the network might not be able to handle. It is known by practitioners that DenseCRF is quite sensitive to its parameters and can easily worsen results. We will use our lucid dreams to handle per-dataset CRF-tuning too, see Sect. 3.2.

We refer to our full $f_{\mathcal{I}+\mathcal{F}}$ system as LucidTracker, and as LucidTracker ${ }^{-}$when no temporal coherency or post-processing steps are used. The usage of $S_{t}$ or model ensemble will be explicitly stated.

\subsection{Training Modalities}

Multiple modalities are available to train a tracker. Trainingfree approaches (e.g. BVS (Maerki et al. 2016), SVT (Wang and Shen 2017)) are fully hand-crafted systems with handtuned parameters, and thus do not require training data. They can be used as-is over different datasets. Supervised methods can also be trained to generate a dataset-agnostic model that can be applied over different datasets. Instead of using a fixed model for all cases, it is also possible to obtain specialized per-dataset models, either via self-supervision (Wang and Gupta 2015; Pathak et al. 2016; Yu et al. 2016; Zhu et al. 2017 ) or by using the first frame annotation of each video in the dataset as training/tuning set. Finally, inspired by traditional box tracking techniques, we also consider adapting the model weights to the specific video at hand, thus obtaining per-video models. Section 5 reports new results over these four training modalities (training-free, dataset-agnostic, perdataset, and per-video).

Our LucidTracker obtains best results when first pretrained on ImageNet, then trained per-dataset using all data from first frame annotations together, and finally fine-tuned per-video for each evaluated sequence. The post-processing DenseCRF stage is automatically tuned per-dataset. The experimental Sect. 5 details the effect of these training stages. Surprisingly, we can obtain reasonable performance even when training from only a single annotated frame (without ImageNet pre-training, i.e. zero pre-training); this results goes against the intuition that convnets require large training data to provide good results.

Unless otherwise stated, we fine-tune per-video models relying solely on the first frame $\mathcal{I}_{0}$ and its annotation $M_{0}$. This is in contrast to traditional techniques (Henriques et al. 2012; Breitenstein et al. 2009; Kristan et al. 2014) which would update the appearance model at each frame $\mathcal{I}_{t}$.

\section{Lucid Data Dreaming}

To train the function $f$ one would think of using ground truth data for $M_{t-1}$ and $M_{t}$ (like (Bertinetto et al. 2016; Caelles et al. 2017; Held et al. 2016)), however such data is expensive to annotate and rare. (Caelles et al. 2017) thus trains on a set of 30 videos ( $\sim 2 \mathrm{k}$ frames $)$ and requires the model to transfer across multiple tests sets. Khoreva et al. (2016) side-steps the need for consecutive frames by generating synthetic masks $M_{t-1}$ from a saliency dataset of $\sim 10 \mathrm{k}$ images with their corresponding mask $M_{t}$. We propose a new data generation strategy to reach better results using only $\sim 100$ individual training frames.

Ideally training data should be as similar as possible to the test data, even subtle differences may affect quality (e.g. training on static images for testing on videos under-performs (Tang et al. 2012)). To ensure our training data is in-domain, we propose to generate it by synthesizing samples from the provided annotated frame (first frame) in each target video. This is akin to "lucid dreaming" as we intentionally "dream" the desired data by creating sample images that are plausible hypothetical future frames of the video. The outcome of this process is a large set of frame pairs in the target domain (2.5k pairs per annotation) with known optical flow and mask annotations, see Fig. 5.

Synthesis Process The target domain for a tracker is the set of future frames of the given video. Traditional data augmentation via small image perturbation is insufficient to cover the expect variations across time, thus a task specific strategy is needed. Across the video the tracked object might change in illumination, deform, translate, be occluded, show different point of views, and evolve on top of a dynamic 


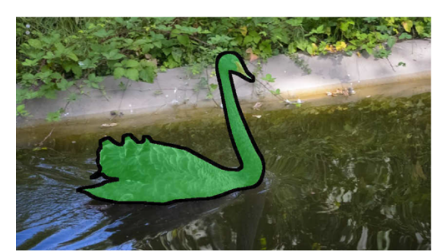

blackswan

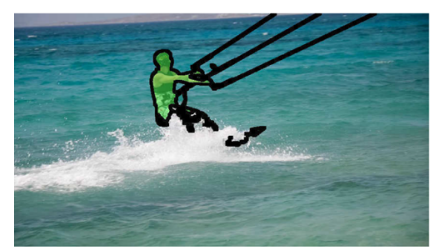

kite-surf

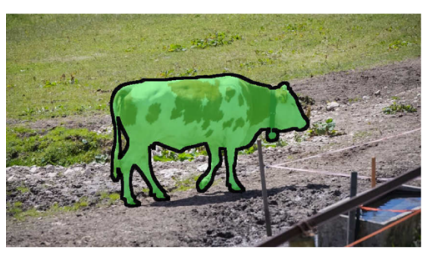

cows

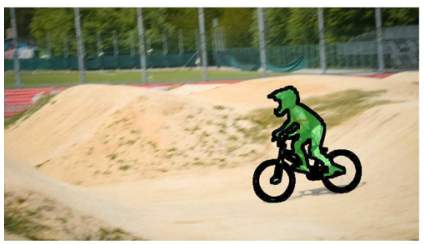

bmx-bumps

Original image $\mathcal{I}_{0}$ and mask annotation $M_{0}$
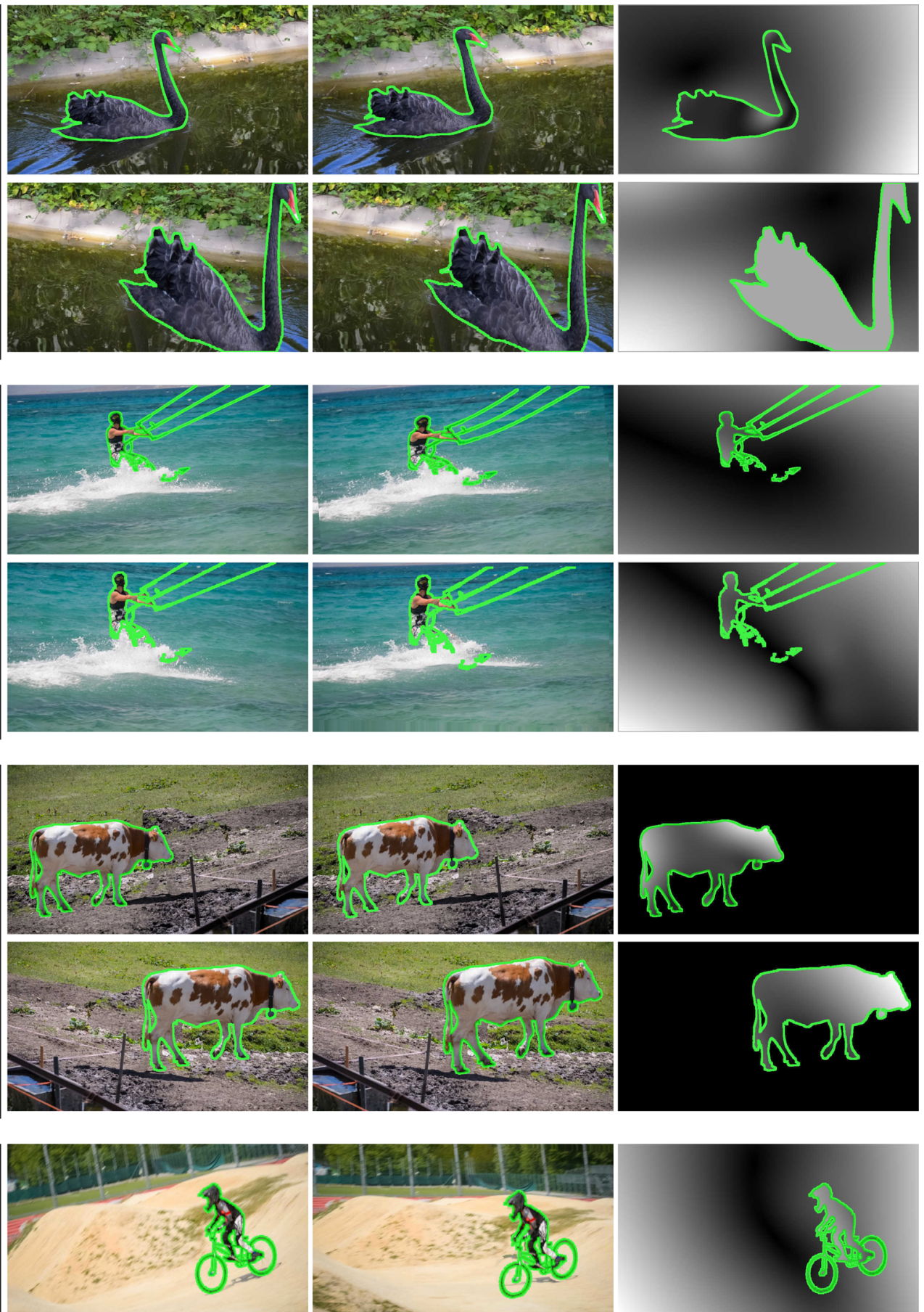

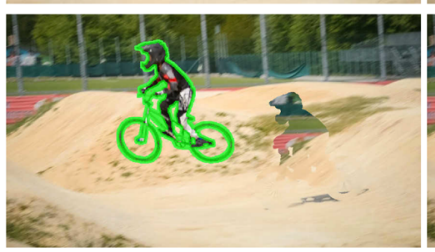

Generated image $\mathcal{I}_{\tau-1}$

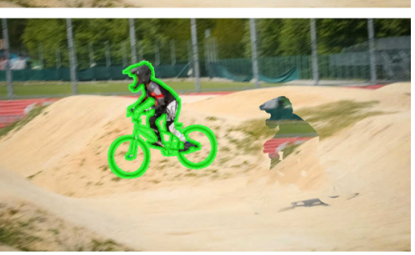

Generated image $\mathcal{I}_{\tau}$

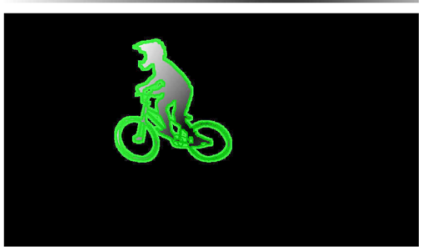

Generated flow magnitude $\left\|\mathcal{F}_{\tau}\right\|$

Fig. 5 Lucid data dreaming examples. From one annotated frame we generate pairs of images $\left(\mathcal{I}_{\tau-1}, \mathcal{I}_{\tau}\right)$ that are plausible future video frames, with known optical flow $\left(\mathcal{F}_{\tau}\right)$ and masks (green boundaries). Note the inpainted background and foreground/background deformations 
background. All of these aspects should be captured when synthesizing future frames. We achieve this by cutting-out the foreground object, in-painting the background, perturbing both foreground and background, and finally recomposing the scene. This process is applied twice with randomly sampled transformation parameters, resulting in a pair of frames $\left(\mathcal{I}_{\tau-1}, \mathcal{I}_{\tau}\right)$ with known pixel-level ground-truth mask annotations $\left(M_{\tau-1}, M_{\tau}\right)$, optical flow $\mathcal{F}_{\tau}$, and occlusion regions. The object position in $\mathcal{I}_{\tau}$ is uniformly sampled, but the changes between $\mathcal{I}_{\tau-1}, \mathcal{I}_{\tau}$ are kept small to mimic the usual evolution between consecutive frames.

In more details, starting from an annotated image:

1. Illumination Changes we globally modify the image by randomly altering saturation $\mathrm{S}$ and value $\mathrm{V}$ (from $\mathrm{HSV}$ colour space) via $x^{\prime}=a \cdot x^{b}+c$, where $a \in 1 \pm 0.05, b \in 1 \pm 0.3$, and $c \in \pm 0.07$.

2. $\mathrm{Fg} / \mathrm{Bg}$ Split the foreground object is removed from the image $\mathcal{I}_{0}$ and a background image is created by inpainting the cut-out area (Criminisi et al. 2004).

3. Object Motion we simulate motion and shape deformations by applying global translation as well as affine and non-rigid deformations to the foreground object. For $\mathcal{I}_{\tau-1}$ the object is placed at any location within the image with a uniform distribution, and in $\mathcal{I}_{\tau}$ with a translation of $\pm 10 \%$ of the object size relative to $\tau-1$. In both frames we apply random rotation $\pm 30^{\circ}$, scaling $\pm 15 \%$ and thin-plate splines deformations (Bookstein 1989) of $\pm 10 \%$ of the object size. 4. Camera Motion We additionally transform the background using affine deformations to simulate camera view changes. We apply here random translation, rotation, and scaling within the same ranges as for the foreground object.

5. Fg/Bg Merge Finally $\left(\mathcal{I}_{\tau-1}, \mathcal{I}_{\tau}\right)$ are composed by blending the perturbed foreground with the perturbed background using Poisson matting (Sun et al. 2004). Using the known transformation parameters we also synthesize ground-truth pixel-level mask annotations $\left(M_{\tau-1}, M_{\tau}\right)$ and optical flow $\mathcal{F}_{\tau}$.

Figure 5 shows example results. Albeit our approach does not capture appearance changes due to point of view, occlusions, nor shadows, we see that already this rough modelling is effective to train our segmentation models.

The number of synthesized images can be arbitrarily large. We generate $2.5 \mathrm{k}$ pairs per annotated video frame. This training data is, by design, in-domain with regard of the target video. The experimental Sect. 5 shows that this strategy is more effective than using thousands of manually annotated images from close-by domains.

The same strategy for data synthesis can be employed for multiple object segmentation task. Instead of manipulating a single object we handle multiple ones at the same time, applying independent transformations to each of them. We model occlusion between objects by adding a random depth ordering obtaining both partial and full occlusions in the training
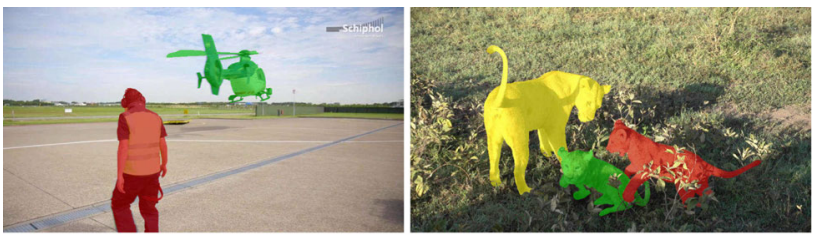

(a) Original image $\mathcal{I}_{0}$ and mask annotation $M_{0}$

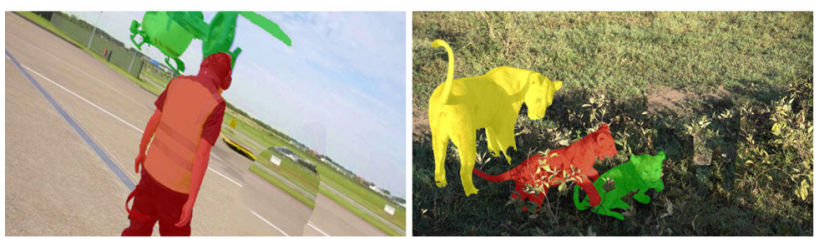

(b) Generated image $\mathcal{I}_{\tau}$ and mask $M_{\tau}$

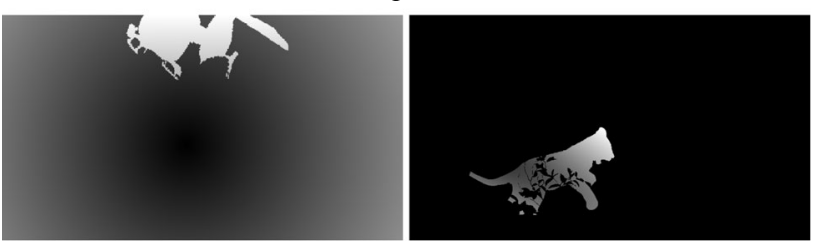

(c) Generated flow magnitude $\left\|\mathcal{F}_{\tau}\right\|$

Fig. 6 Lucid data dreaming examples with multiple objects. From one annotated frame we generate a plausible future video frame $\left(\mathcal{I}_{\tau}\right)$, with known optical flow $\left(\mathcal{F}_{\tau}\right)$ and mask $\left(M_{\tau}\right)$

set. Including occlusions in the lucid dreams allows to better handle plausible interactions of objects in the future frames. See Fig. 6 for examples of the generated data.

\section{Single Object Segmentation Results}

We present here a detailed empirical evaluation on three different datasets for the single object segmentation task: given a first frame labelled with the foreground object mask, the goal is to find the corresponding object pixels in future frames. (Section 6 will discuss the multiple objects case.)

\subsection{Experimental Setup}

Datasets We evaluate our method on three video object segmentation datasets: DAVIS 16 (Perazzi et al. 2016), YouTubeObjects (Prest et al. 2012; Jain and Grauman 2014), and $\mathrm{SegTrack}_{\mathrm{v} 2}$ (Li et al. 2013). The goal is to track an object through all video frames given an object mask in the first frame. These three datasets provide diverse challenges with a mix of high and low resolution web videos, single or multiple salient objects per video, videos with flocks of similar looking instances, longer $(\sim 400$ frames $)$ and shorter $(\sim 10$ frames) sequences, as well as the usual video segmentation challenges such as occlusion, fast motion, illumination, view point changes, elastic deformation, etc. 
The DAVIS 16 (Perazzi et al. 2016) video segmentation benchmark consists of 50 full-HD videos of diverse object categories with all frames annotated with pixel-level accuracy, where one single or two connected moving objects are separated from the background. The number of frames in each video varies from 25 to 104.

YouTubeObjects (Prest et al. 2012; Jain and Grauman 2014) includes web videos from 10 object categories. We use the subset of 126 video sequences with mask annotations provided by Jain and Grauman (2014) for evaluation, where one single object or a group of objects of the same category are separated from the background. In contrast to DAVIS 16 these videos have a mix of static and moving objects. The number of frames in each video ranges from 2 to 401 .

SegTrack 2 Li et al. (2013) consists of 14 videos with multiple object annotations for each frame. For videos with multiple objects each object is treated as a separate problem, resulting in 24 sequences. The length of each video varies from 21 to 279 frames. The images in this dataset have low resolution and some compression artefacts, making it hard to track the object based on its appearance.

The main experimental work is done on DAVIS 16 , since it is the largest densely annotated dataset out of the three, and provides high quality/high resolution data. The videos for this dataset were chosen to represent diverse challenges, making it a good experimental playground.

We additionally report on the two other datasets as complementary test set results.

Evaluation Metric To measure the accuracy of video object segmentation we use the mean intersection-over-union overlap (mIoU) between the per-frame ground truth object mask and the predicted segmentation, averaged across all video sequences. We have noticed disparate evaluation procedures used in previous work, and we report here a unified evaluation across datasets. When possible, we re-evaluated certain methods using results provided by their authors. For all three datasets we follow the DAVIS 16 evaluation protocol, excluding the first frame from evaluation and using all other frames from the video sequences, independent of object presence in the frame.

Training Details For training all the models we use SGD with mini-batches of 10 images and a fixed learning policy with initial learning rate of $10^{-3}$. The momentum and weight decay are set to 0.9 and $5 \times 10^{-4}$, respectively.

Models using pre-training are initialized with weights trained for image classification on ImageNet (Simonyan and Zisserman 2015). We then train per-dataset for 40k iterations with the RGB+Mask branch $f_{\mathcal{I}}$ and for 20k iterations for the Flow+Mask $f_{\mathcal{F}}$ branch. When using a single stream architecture (Sect. 5.4.3), we use 40k iterations.

Models without ImageNet pre-training are initialized using the Xavier (also known as Glorot) random weight ini- tialization strategy (Glorot and Bengio 2010). (The weights are initialized as random draws from a truncated normal distribution with zero mean and standard deviation calculated based on the number of input and output units in the weight tensor, see Glorot and Bengio (2010) for details). The perdataset training needs to be longer, using $100 \mathrm{k}$ iterations for the $f_{\mathcal{I}}$ branch and $40 \mathrm{k}$ iterations for the $f_{\mathcal{F}}$ branch.

For per-video fine-tuning $2 \mathrm{k}$ iterations are used for $f_{\mathcal{I}}$. To keep computing cost lower, the $f_{\mathcal{F}}$ branch is kept fix across videos.

All training parameters are chosen based on DAVIS 16 results. We use identical parameters on YouTubeObjects and SegTrack $_{\mathrm{v} 2}$, showing the generalization of our approach.

It takes $\sim 3.5 \mathrm{~h}$ to obtain each per-video model, including data generation, per-dataset training, per-video fine-tuning and per-dataset grid search of CRF parameters (averaged over DAVIS $_{16}$, amortising the per-dataset training time over all videos). At test time our LucidTracker runs at $\sim 5 \mathrm{~s}$ per frame, including the optical flow estimation with FlowNet2.0 (Ilg et al. 2017) ( 0.5 s) and CRF post-processing (Krähenbühl and Koltun 2011) ( 2s).

\subsection{Key Results}

Table 1 presents our main result and compares it to previous work. Our full system, LucidTracker, provides the best video segmentation quality across three datasets while being trained on each dataset using only one frame per video (50 frames for DAVIS 16,126 for YouTubeObjects, 24 for SegTrack $_{\mathrm{v} 2}$ ), which is $20 \times-1000 \times$ less than the top competing methods. Ours is the first method to reach $>75 \mathrm{mIoU}$ on all three datasets.

Oracles and Baselines Grabcut oracle computes grabcut (Rother et al. 2004) using the ground truth bounding boxes (box oracle). This oracle indicates that on the considered datasets separating foreground from background is not easy, even if a perfect box-level tracker was available.

We provide three additional baselines. "Saliency" corresponds to using the generic (training-free) saliency method EQCut (Aytekin et al. 2015) over the RGB image $\mathcal{I}_{t}$. "Flow saliency" does the same, but over the optical flow magnitude $\left\|\mathcal{F}_{t}\right\|$. Results indicate that the objects being tracked are not particularly salient in the image. On DAVIS 16 motion saliency is a strong signal but not on the other two datasets. Saliency methods ignore the first frame annotation provided for the task. We also consider the "Mask warping" baseline which uses optical flow to propagate the mask estimate from $t$ to $t+1$ via simple warping $M_{t}=w\left(M_{t-1}, \mathcal{F}_{t}\right)$. The bad results of this baseline indicate that the high quality flow (Ilg et al. 2017) that we use is by itself insufficient to solve the video object segmentation task, and that indeed our proposed convnet does the heavy lifting. 
Table 1 Comparison of video object segmentation results across three datasets. Our LucidTracker consistently improves over previous results, see Sect. 5.2

\begin{tabular}{|c|c|c|c|c|c|}
\hline \multirow[t]{2}{*}{ Method } & \multirow[t]{2}{*}{ \# Training images } & \multirow{2}{*}{$\begin{array}{l}\text { Flow } \\
\mathcal{F}\end{array}$} & \multicolumn{3}{|c|}{$\underline{\text { Dataset, } \mathrm{mIoU}}$} \\
\hline & & & DAVIS $_{16}$ & YoutbObjs & SegTrck $_{\mathrm{v} 2}$ \\
\hline Box oracle (Khoreva et al. 2016) & 0 & $x$ & 45.1 & 55.3 & 56.1 \\
\hline Grabcut oracle (Khoreva et al. 2016) & 0 & $x$ & 67.3 & 67.6 & 74.2 \\
\hline \multicolumn{6}{|l|}{ Ignores 1st frame annotation } \\
\hline Saliency & 0 & $x$ & 32.7 & 40.7 & 22.2 \\
\hline NLC (Faktor and Irani 2014) & 0 & $\checkmark$ & 64.1 & - & \\
\hline TRS (Xiao and Lee 2016) & 0 & $\checkmark$ & - & - & 69.1 \\
\hline MP-Net (Tokmakov et al. 2016) & $\sim 22.5 \mathrm{k}$ & $\checkmark$ & 69.7 & - & - \\
\hline Flow saliency & 0 & $\checkmark$ & 70.7 & 36.3 & 35.9 \\
\hline FusionSeg (Jain et al. 2017) & $\sim 95 \mathrm{k}$ & $\checkmark$ & 71.5 & 67.9 & - \\
\hline LVO (Tokmakov et al. 2017) & $\sim 35 \mathrm{k}$ & $\checkmark$ & 75.9 & - & 57.3 \\
\hline PDB (Song et al. 2018) & $\sim 18 \mathrm{k}$ & $x$ & 77.2 & - & - \\
\hline \multicolumn{6}{|l|}{ Uses 1st frame annotation } \\
\hline Mask warping & 0 & $\checkmark$ & 32.1 & 43.2 & 42.0 \\
\hline FCP (Perazzi et al. 2015) & 0 & $\checkmark$ & 63.1 & - & - \\
\hline BVS (Maerki et al. 2016) & 0 & $x$ & 66.5 & 59.7 & 58.4 \\
\hline N15 (Nagaraja et al. 2015) & 0 & $\checkmark$ & - & - & 69.6 \\
\hline ObjFlow (Tsai et al. 2016) & 0 & $\sqrt{ }$ & 71.1 & 70.1 & 67.5 \\
\hline STV (Wang and Shen 2017) & 0 & $\checkmark$ & 73.6 & - & - \\
\hline VPN (Jampani et al. 2016) & $\sim 2.3 \mathrm{k}$ & $x$ & 75.0 & - & - \\
\hline OSVOS (Caelles et al. 2017) & $\sim 2.3 \mathrm{k}$ & $x$ & 79.8 & 72.5 & 65.4 \\
\hline MaskTrack (Khoreva et al. 2016) & $\sim 11 \mathrm{k}$ & $\checkmark$ & 80.3 & 72.6 & 70.3 \\
\hline PReMVOS (Luiten and Voigtlaender 2018) & $\sim 145 \mathrm{k}$ & $\checkmark$ & 84.9 & - & - \\
\hline OnAVOS (Voigtlaender and Leibe 2017b) & $\sim 120 \mathrm{k}$ & $x$ & 86.1 & - & - \\
\hline VideoGCRF (Chandra et al. 2018) & $\sim 120 \mathrm{k}$ & $x$ & 86.5 & - & - \\
\hline LucidTracker & 24-126 & $\checkmark$ & 86.6 & 77.3 & 78.0 \\
\hline
\end{tabular}

Numbers in italic are reported on subsets of DAVIS 16 and in bold are the best numbers overall
The large fluctuation of the relative baseline results across the three datasets empirically confirms that each of them presents unique challenges.

Comparison Compared to flow propagation methods such as BVS, N15, ObjFlow, and STV, we obtain better results because we build per-video a stronger appearance model of the tracked object (embodied in the fine-tuned model). Compared to convnet learning methods such as VPN, OSVOS, MaskTrack, OnAVOS, we require significantly less training data, yet obtain better results.

Figure 7 provides qualitative results of LucidTracker across three different datasets. Our system is robust to various challenges present in videos. It handles well camera view changes, fast motion, object shape deformation, outof-view scenarios, multiple similar looking objects and even low quality video. We provide a detailed error analysis in Sect. 5.5.
Conclusion We show that top results can be obtained while using less training data. This shows that our lucid dreams leverage the available training data better. We report top results for this task while using only 24-126 training frames.

\subsection{Ablation Studies}

In this section we explore in more details how the different ingredients contribute to our results.

\subsubsection{Effect of Training Modalities}

Table 2 compares the effect of different ingredients in the Luciatracker ${ }^{-}$training. Results are obtained using RGB and flow, with warping, no CRF, and no temporal coherency; $M_{t}=f\left(\mathcal{I}_{t}, w\left(M_{t-1}, \mathcal{F}_{t}\right)\right)$. 

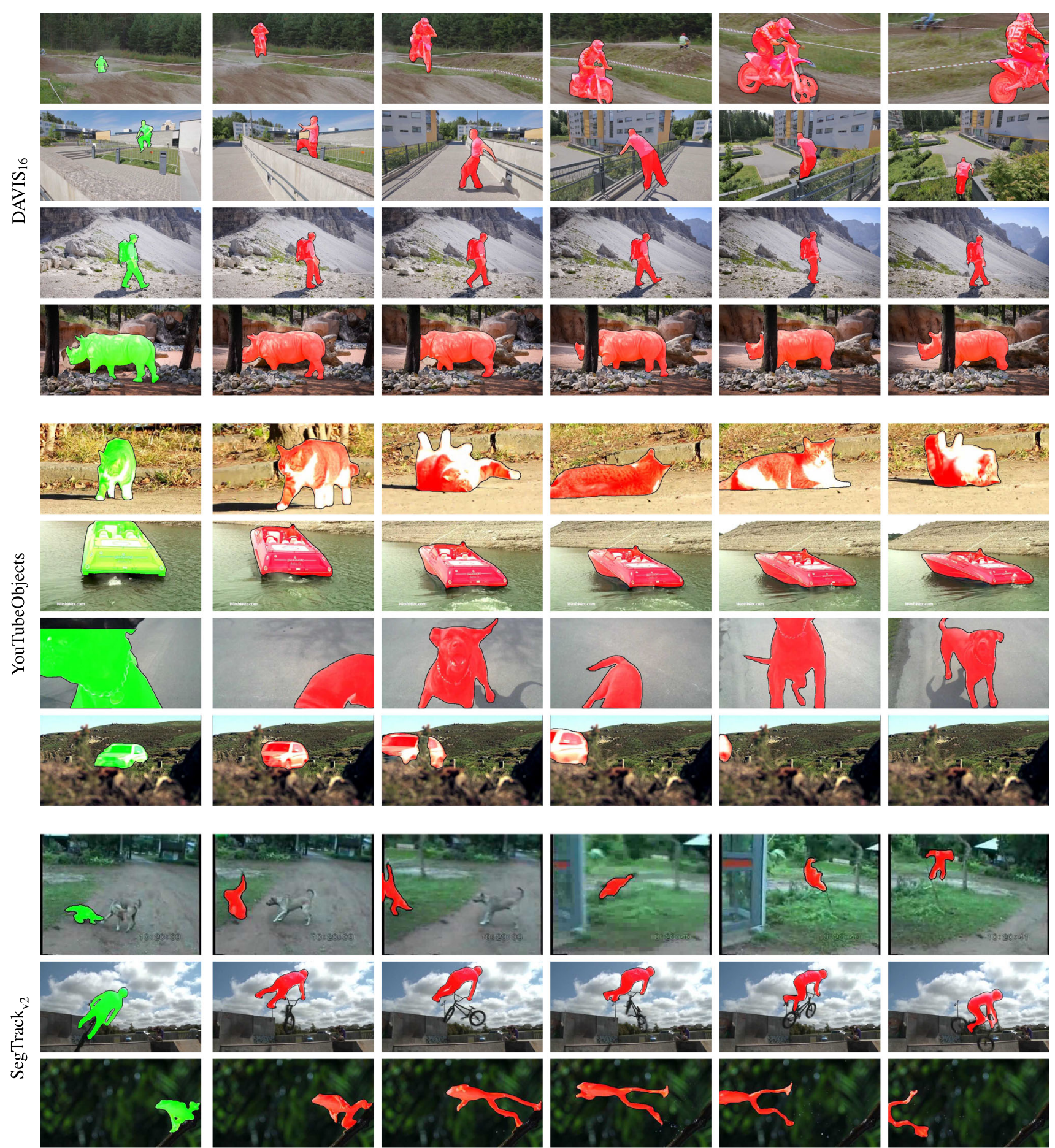

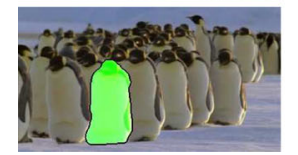

1st frame, GT segment
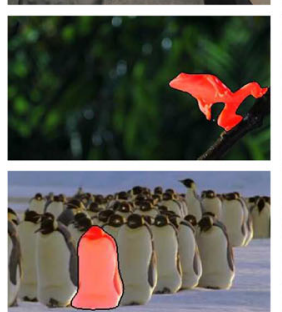

$20 \%$
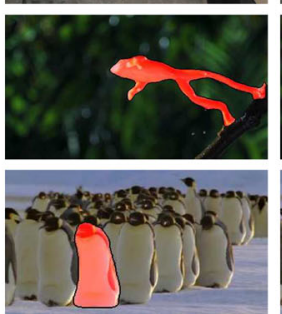

$40 \%$
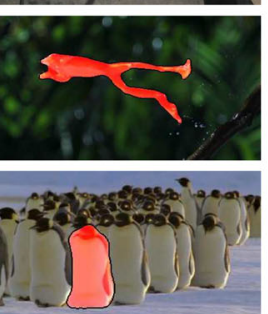

$60 \%$
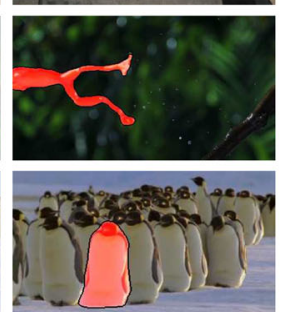

$80 \%$
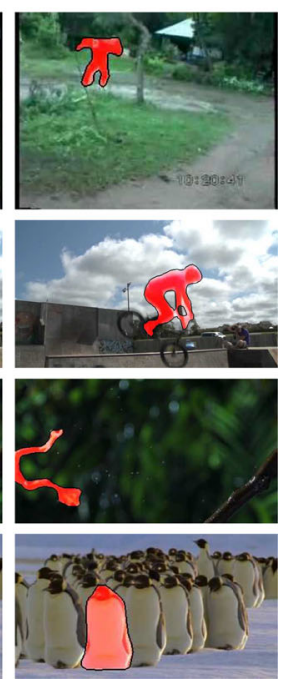

$100 \%$

Fig. 7 LucidTracker single object segmentation qualitative results. Frames sampled along the video duration (e.g. 50\%: video middle point). Our model is robust to various challenges, such as view changes, fast motion, shape deformations, and out-of-view scenarios 
Table 2 Ablation study of training modalities. ImageNet pre-training and per-video tuning provide additional improvement over per-dataset training. Even with one frame annotation for only per-video tuning we obtain good performance. See Sect. 5.3.1
Table 3 Ablation study of flow ingredients. Flow complements image only results, with large fluctuations across datasets. See Sect. 5.3.2

\begin{tabular}{|c|c|c|c|c|c|c|}
\hline \multirow[t]{2}{*}{ Variant } & \multirow{2}{*}{$\begin{array}{l}\text { ImgNet } \\
\text { pre-train. }\end{array}$} & \multirow{2}{*}{$\begin{array}{l}\text { Per-dataset } \\
\text { training }\end{array}$} & \multirow{2}{*}{$\begin{array}{l}\text { Per-video } \\
\text { fine-tun. }\end{array}$} & \multicolumn{3}{|c|}{ Dataset, mIoU } \\
\hline & & & & DAVIS $_{16}$ & YoutbObjs & SegTrck $_{\mathrm{V} 2}$ \\
\hline LucidTracker $^{-}$ & $\checkmark$ & $\checkmark$ & $\checkmark$ & 83.7 & 76.2 & 76.8 \\
\hline (no ImgNet) & $x$ & $\checkmark$ & $\checkmark$ & 82.0 & 74.3 & 71.2 \\
\hline \multirow[t]{2}{*}{ No per-video tuning } & $\checkmark$ & $\checkmark$ & $x$ & 82.7 & 72.3 & 71.9 \\
\hline & $x$ & $\checkmark$ & $x$ & 78.4 & 69.7 & 68.2 \\
\hline \multirow[t]{2}{*}{ Only per-video tuning } & $\checkmark$ & $x$ & $\checkmark$ & 79.4 & - & 70.4 \\
\hline & $x$ & $x$ & $\checkmark$ & 80.5 & - & 66.8 \\
\hline
\end{tabular}

Numbers in italic are reported on subsets of DAVIS 16 and in bold are the best numbers overall

\begin{tabular}{|c|c|c|c|c|c|c|}
\hline \multirow[t]{2}{*}{ Variant } & \multirow[t]{2}{*}{$\mathcal{I}$} & \multirow[t]{2}{*}{$\mathcal{F}$} & \multirow{2}{*}{$\begin{array}{l}\text { warp. } \\
w\end{array}$} & \multicolumn{3}{|c|}{ Dataset, mIoU } \\
\hline & & & & DAVIS $_{16}$ & YoutbObjs & $\operatorname{SegTrck}_{\mathrm{v} 2}$ \\
\hline LucidTracker & $\sqrt{ }$ & $\sqrt{ }$ & $\checkmark$ & 86.6 & 77.3 & 78.0 \\
\hline LucidTracker $^{-}$ & $\sqrt{ }$ & $\sqrt{ }$ & $\sqrt{ }$ & 83.7 & 76.2 & 76.8 \\
\hline No warping & $\sqrt{ }$ & $\sqrt{ }$ & $x$ & 82.0 & 74.6 & 70.5 \\
\hline No OF & $\sqrt{ }$ & $x$ & $x$ & 78.0 & 74.7 & 61.8 \\
\hline OF only & $x$ & $\sqrt{ }$ & $\sqrt{ }$ & 74.5 & 43.1 & 55.8 \\
\hline
\end{tabular}

Bold are the best numbers overall
Training from a Single Frame In the bottom row ("only per-video tuning"), the model is trained per-video without ImageNet pre-training nor per-dataset training, i.e. using a single annotated training frame. Our network is based on VGG16 (Chen et al. 2016) and contains $\sim 20 \mathrm{M}$ parameters, all effectively learnt from a single annotated image that is augmented to become $2.5 \mathrm{k}$ training samples (see Sect. 4). Even with such minimal amount of training data, we still obtain a surprisingly good performance (compare 80.5 on DAVIS $_{16}$ to others in Table 1). This shows how effective is, by itself, the proposed training strategy based on lucid dreaming of the data.

Pre-training \& Fine-Tuning We see that ImageNet pretraining does provide $2-5 \%$ point improvement (depending on the dataset of interest; e.g. $82.0 \rightarrow 83.7 \mathrm{mIoU}$ on DAVIS $_{16}$ ). Per-video fine-tuning (after doing per-dataset training) provides an additional $1-2 \%$ point gain (e.g. $82.7 \rightarrow 83.7 \mathrm{mIoU}$ on DAVIS $\left.{ }_{16}\right)$. Both ingredients clearly contribute to the segmentation results.

Note that training a model using only per-video tuning takes about one full GPU day per video sequence; making these results insightful but not decidedly practical.

Preliminary experiments evaluating on DAVIS $_{16}$ the impact of the different ingredients of our lucid dreaming data generation showed, depending on the exact setup, 3$10 \%$ mIoU points fluctuations between a basic version (e.g. without non-rigid deformations nor scene re-composition) and the full synthesis process described in Sect. 4. Having a sophisticated data generation process directly impacts the segmentation quality.

Conclusion Surprisingly, we discovered that per-video training from a single annotated frame provides already much of the information needed for the video object segmentation task. Additionally using ImageNet pre-training, and perdataset training, provide complementary gains.

\subsubsection{Effect of Optical Flow}

Table 3 shows the effect of optical flow on LucidTracker results. Comparing our full system to the "No OF" row, we see that the effect of optical flow varies across datasets, from minor improvement in YouTubeObjects, to major difference in SegTrack $\mathrm{v}_{\mathrm{v} 2}$. In this last dataset, using mask warping is particularly useful too. We additionally explored tuning the optical flow stream per-video, which resulted in a minor improvement $(83.7 \rightarrow 83.9$ mIoU on DAVIS 16 ).

Our "No OF" results can be compared to OSVOS (Caelles et al. 2017) which does not use optical flow. However OSVOS uses a per-frame mask post-processing based on a boundary detector (trained on further external data), which provides $\sim 2 \%$ point gain. Accounting for this, our "No OF" (and no CRF, no temporal coherency) result matches theirs on DAVIS $_{16}$ and YouTubeObjects despite using significantly 
Table 4 Effect of optical flow estimation

Table 5 Effect of CRF tuning (LucidTracker without temporal coherency). Without the automated per-dataset tuning DenseCRF will under-perform

\begin{tabular}{lllll}
\hline Variant & Optical flow & Dataset, mIoU & \\
\cline { 3 - 5 } & & DAVIS $_{16}$ & YoutbObjs & SegTrck $_{\text {v2 }}$ \\
\hline LucidTracker $^{-}$ & FlowNet2.0 & $\mathbf{8 3 . 7}$ & $\mathbf{7 6 . 2}$ & $\mathbf{7 6 . 8}$ \\
& EpicFlow & 80.2 & 71.3 & 67.0 \\
& No flow & 78.0 & 74.7 & 61.8 \\
\hline No ImageNet pre-training & FlowNet2.0 & 82.0 & 74.3 & 71.2 \\
& EpicFlow & 80.0 & 72.3 & 68.8 \\
& No flow & 76.7 & 71.4 & 63.0 \\
\hline
\end{tabular}

Bold are the best numbers overall

\begin{tabular}{lllll}
\hline Method & CRF parameters & Dataset, mIoU & \\
\cline { 3 - 5 } & & DAVIS $_{16}$ & YoutbObjs & SegTrck $_{\mathrm{v} 2}$ \\
\hline LucidTracker $^{-}$ & - & 83.7 & 76.2 & 76.8 \\
LucidTracker & Default & 84.2 & 75.5 & 72.2 \\
LucidTracker & Tuned per-dataset & $\mathbf{8 4 . 8}$ & $\mathbf{7 6 . 2}$ & $\mathbf{7 7 . 6}$ \\
\hline
\end{tabular}

Bold are the best numbers overall

less training data (see Table 1, e.g. 79.8-2 $\approx 78.0$ on DAVIS $_{16}$ ).

Table 4 shows the effect of using different optical flow estimation methods. For LucidTracker results, FlowNet2.0 (Ilg et al. 2017) was employed. We also explored using EpicFlow (Revaud et al. 2015), as in Khoreva et al. (2016). Table 4 indicates that employing a robust optical flow estimation across datasets is crucial to the performance (FlowNet2.0 provides $\sim 1.5-15$ points gain on each dataset). We found EpicFlow to be brittle when going across different datasets, providing improvement for DAVIS 16 and SegTrack $_{\mathrm{v} 2}(\sim$ $2-5$ points gain), but underperforming for YouTubeObjects $(74.7 \rightarrow 71.3 \mathrm{mIoU})$.

Conclusion The results show that flow provides a complementary signal to RGB image only and having a robust optical flow estimation across datasets is crucial. Despite its simplicity our fusion strategy $\left(f_{\mathcal{I}}+f_{\mathcal{F}}\right)$ provides gains on all datasets, and leads to competitive results.

\subsubsection{Effect of CRF Tuning}

As a final stage of our pipeline, we refine the generated mask using DenseCRF (Krähenbühl and Koltun 2011) per frame. This captures small image details that the network might have missed. It is known by practitioners that DenseCRF is quite sensitive to its parameters and can easily worsen results. We use our lucid dreams to enable automatic per-dataset CRFtuning.

Following Chen et al. (2016) we employ grid search scheme for tuning CRF parameters. Once the per-dataset model is trained, we apply it over a subset of its training set (5 random images from the lucid dreams per video sequence), apply DenseCRF with the given parameters over this output, and then compare to the lucid dream ground truth.

The impact of the tuned parameter of DenseCRF postprocessing is shown in Table 5 and Fig. 8. Table 5 indicates that without per-dataset tuning DenseCRF is underperforming. Our automated tuning procedure allows to obtain consistent gains without the need for case-by-case manual tuning.

Conclusion Using default DenseCRF parameters would degrade performance. Our lucid dreams enable automatic per-dataset CRF-tuning which allows to further improve the results.

\subsection{Additional Experiments}

Other than adding or removing ingredients, as in Sect. 5.3, we also want to understand how the training data itself affects the obtained results.

\subsubsection{Generalization Across Videos}

Table 6 explores the effect of segmentation quality as a function of the number of training samples. To see more directly the training data effects we use a base model with RGB image $\mathcal{I}_{t}$ only (no flow $\mathcal{F}$, no CRF, no temporal coherency), and per-dataset training (no ImageNet pre-training, no pervideo fine-tuning). We evaluate on two disjoint subsets of 15 DAVIS $_{16}$ videos each, where the first frames for per-dataset training are taken from only one subset. The reported numbers are thus comparable within Table 6 , but not across to the other tables in the paper. Table 6 reports results with varying number of training videos and with/without including the 

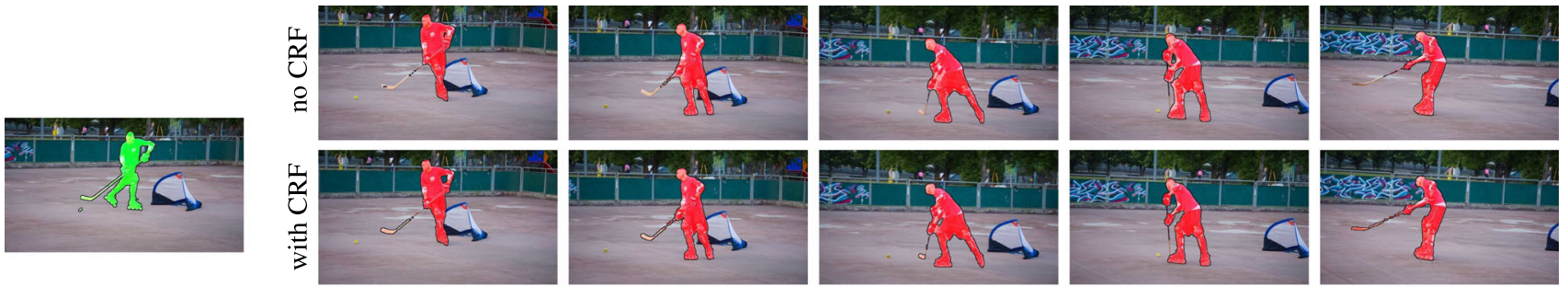

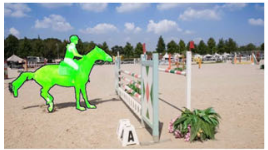

1st frame, GT segment
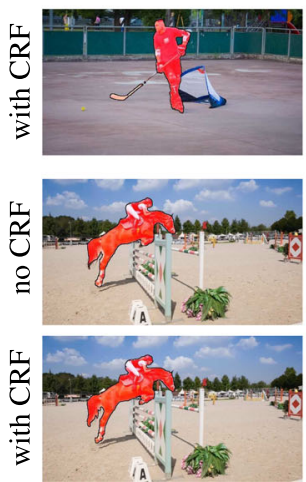

$20 \%$
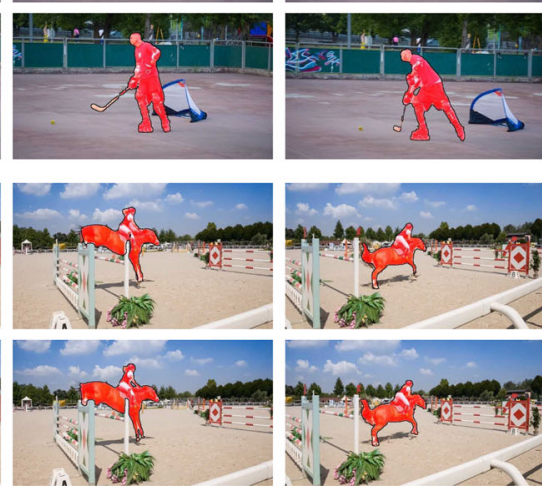

$40 \%$

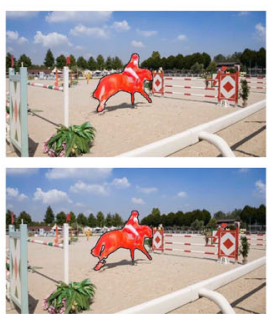

$80 \%$

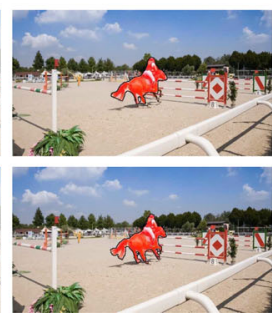

$100 \%$

Fig. 8 Effect of CRF tuning. The shown DAVIS ${ }_{16}$ videos have the highest margin between with and without CRF post-processing (based on mIoU over the video)

Table 6 Varying the number of training videos. A smaller training set closer to the target domain is better than a larger one. See Sect. 5.4.1

\begin{tabular}{lccc}
\hline Training set & $\begin{array}{l}\text { \# Training } \\
\text { videos }\end{array}$ & $\begin{array}{l}\text { \# Frames } \\
\text { per video }\end{array}$ & mIoU \\
\hline Includes 1st frames from test set & 1 & 1 & 78.3 \\
& 2 & 1 & 75.4 \\
& 15 & 1 & 68.7 \\
Excludes 1st frames from test set & 30 & 1 & 65.4 \\
& 30 & 1 & 74.3 \\
\hline & 2 & 1 & 11.6 \\
& 15 & 1 & 36.4 \\
& 30 & 2 & 41.7 \\
\hline
\end{tabular}

first frames of each test video for per-dataset training. When excluding the test set first frames, the image frames used for training are separate from the test videos; and we are thus operating across (related) domains. When including the test set first frames, we operate in the usual LucidTracker mode, where the first frame from each test video is used to build the per-dataset training set.

Comparing the top and bottom parts of the table, we see that when the annotated images from the test set video sequences are not included, segmentation quality drops drastically (e.g. $68.7 \rightarrow 36.4 \mathrm{mIoU}$ ). Conversely, on subset of videos for which the first frame annotation is used for training, the quality is much higher and improves as the training samples become more and more specific (in-domain) to the target video (65.4 $\rightarrow 78.3 \mathrm{mIoU})$. Adding extra videos for training does not improve the performance. It is better $(68.7 \rightarrow 78.3 \mathrm{mIoU})$ to have 15 models each trained and evaluated on a single video (row top-1-1) than having one model trained over 15 test videos (row top-15-1).
Training with an additional frame from each video (we added the last frame of each train video) significantly boosts the resulting within-video quality (e.g. row top-30-2 $65.4 \rightarrow$ $74.3 \mathrm{mIoU}$ ), because the training samples cover better the test domain.

Conclusion These results show that, when using RGB information $\left(\mathcal{I}_{t}\right)$, increasing the number of training videos does not improve the resulting quality of our system. Even within a dataset, properly using the training sample(s) from within each video matters more than collecting more videos to build a larger training set.

\subsubsection{Generalization Across Datasets}

Section 5.4.1 has explored the effect of changing the volume of training data within one dataset, Table 7 compares results when using different datasets for training. Results are obtained using a base model with RGB and flow $\left(M_{t}=\right.$ $f\left(\mathcal{I}_{t}, M_{t-1}\right)$, no warping, no CRF, no temporal coherency), 
Table 7 Generalization across datasets. We observe a significant quality gap between training from the target videos, versus training from other datasets; see Sect. 5.4.2

\begin{tabular}{lllll}
\hline Training set & \multicolumn{2}{l}{ Dataset, mIoU } & Mean \\
\cline { 2 - 4 } & DAVIS $_{16}$ & YoutbObjs & SegTrck $_{\mathrm{v} 2}$ & \\
\hline DAVIS $_{16}$ & $\underline{80.9}$ & 50.9 & 46.9 & 59.6 \\
YoutbObjs & 67.0 & $\underline{71.5}$ & 52.0 & 63.5 \\
SegTrack 2 & 56.0 & 52.2 & $\underline{66.4}$ & 58.2 \\
\hline Best & $\mathbf{8 0 . 9}$ & $\mathbf{7 1 . 5}$ & $\mathbf{6 6 . 4}$ & $\mathbf{7 2 . 9}$ \\
Second best & 67.0 & 52.2 & 52.0 & 57.1 \\
\hline All-in-one & 71.9 & 70.7 & 60.8 & 67.8 \\
\hline Results & & & & 6
\end{tabular}

Results with underline are the best per dataset, in bold are the best numbers overall, and in italic are the second best per dataset (ignoring all-in-one setup)

ImageNet pre-training, per-dataset training, and no per-video tuning to accentuate the effect of the training dataset.

The best performance is obtained when training on the first frames of the target set. There is a noticeable $\sim 10 \%$ points drop when moving to the second best choice (e.g. $80.9 \rightarrow 67.0$ for DAVIS $_{16}$ ). Interestingly, when putting all the datasets together for training ("all-in-one" row, a datasetagnostic model) the results degrade, reinforcing the idea that "just adding more data" does not automatically make the performance better.

Conclusion Best results are obtained when using training data that focuses on the test video sequences, using similar datasets or combining multiple datasets degrades the performance for our system.

\subsubsection{Experimenting with the Convnet Architecture}

Section 3.1 and Fig. 3 described two possible architectures to handle $\mathcal{I}_{t}$ and $\mathcal{F}_{t}$. Previous experiments are all based on the two streams architecture.

Table 8 compares two streams versus one stream, where the network to accepts 5 input channels (RGB + previous mask + flow magnitude) in one stream: $M_{t}=f_{\mathcal{I}+\mathcal{F}}\left(\mathcal{I}_{t}\right.$, $\left.\mathcal{F}_{t}, w\left(M_{t-1}, \mathcal{F}_{t}\right)\right)$. Results are obtained using a base model with RGB and optical flow (no warping, no CRF, no temporal coherency), ImageNet pre-training, per-dataset training, and no per-video tuning.
We observe that both one stream and two stream architecture with naive averaging perform on par. Using a one stream network makes the training more affordable and allows more easily to expand the architecture with additional input channels.

Conclusion The lighter one stream network performs as well as a network with two streams. We will thus use the one stream architecture in Sect. 6.

\subsection{Error Analysis}

Table 9 presents an expanded evaluation on DAVIS 16 using evaluation metrics proposed in Perazzi et al. (2016). Three measures are used: region similarity in terms of intersection over union $(\mathrm{J})$, contour accuracy ( $\mathrm{F}$, higher is better), and temporal instability of the masks ( $\mathrm{T}$, lower is better). We outperform the competitive methods (Khoreva et al. 2016; Caelles et al. 2017) on all three measures.

Table 10 reports the per-attribute based evaluation as defined in DAVIS 16 . LucidTracker is best on all 15 video attribute categories. This shows that our LucidTracker can handle the various video challenges present in DAVIS 16 .

We present the per-sequence and per-frame results of LucidTracker over DAVIS 16 in Fig. 9. On the whole we observe that the proposed approach is quite robust, most video sequences reach an average performance above 80 mIoU.

However, by looking at per-frame results for each video (blue dots in Fig. 9) one can see several frames where our approach has failed (IoU less than 50) to correctly track the object. Investigating closely those cases we notice conditions where LucidTracker is more likely to fail. The same behaviour was observed across all three datasets. A few representatives of failure cases are visualized in Fig. 10.

Since we are using only the mask annotation of the first frame for training the tracker, a clear failure case is caused by dramatic view point changes of the object from its first frame appearance, as in row 5 of Fig. 10. Performing online adaptation every certain time step while exploiting the previous frame segments for data synthesis and marking unsure regions as ignore for training, similarly to Voigtlaender and Leibe (2017b), might resolve the potential problems caused by relying only on the first frame mask. The proposed approach also under-performs when recovering from occlu-
Table 8 Experimenting with the convnet architecture. See Sect. 5.4 .3

\begin{tabular}{lllll}
\hline Architecture & ImgNet pre-train. & Per-dataset training & Per-video fine-tun. & $\begin{array}{l}\text { DAVIS } \\
\text { mIoU }\end{array}$ \\
\hline Two streams & $\checkmark$ & $\checkmark$ & $\boldsymbol{X}$ & $\mathbf{8 0 . 9}$ \\
One stream & $\checkmark$ & $\checkmark$ & $\boldsymbol{X}$ & 80.3 \\
\hline
\end{tabular}

Bold is the best number overall 
Table 9 Comparison of video object segmentation results on DAVIS 16 benchmark. Our LucidTracker improves over previous results

\begin{tabular}{|c|c|c|c|c|c|c|c|c|c|}
\hline \multirow[t]{3}{*}{ Method } & \multirow{3}{*}{$\begin{array}{l}\text { \# Training } \\
\text { images }\end{array}$} & \multirow{3}{*}{ Flow $\mathcal{F}$} & \multicolumn{7}{|l|}{ DAVIS $_{16}$} \\
\hline & & & \multicolumn{3}{|c|}{$\underline{\text { Region, } J}$} & \multicolumn{3}{|c|}{ Boundary, $F$} & \multirow{2}{*}{$\begin{array}{l}\text { Temporal stability, } T \\
\text { Mean } \downarrow\end{array}$} \\
\hline & & & Mean $\uparrow$ & Recall $\uparrow$ & Decay $\downarrow$ & Mean $\uparrow$ & Recall $\uparrow$ & Decay $\downarrow$ & \\
\hline Box oracle (Khoreva et al. 2016) & 0 & $x$ & 45.1 & 39.7 & -0.7 & 21.4 & 6.7 & 1.8 & 1.0 \\
\hline Grabcut oracle (Khoreva et al. 2016) & 0 & $x$ & 67.3 & 76.9 & 1.5 & 65.8 & 77.2 & 2.9 & 34.0 \\
\hline \multicolumn{10}{|l|}{ Ignores 1 st frame annotation } \\
\hline Saliency & 0 & $x$ & 32.7 & 22.6 & -0.2 & 26.9 & 10.3 & 0.9 & 32.8 \\
\hline NLC (Faktor and Irani 2014) & 0 & $\checkmark$ & 64.1 & 73.1 & 8.6 & 59.3 & 65.8 & 8.6 & 35.8 \\
\hline MP-Net (Tokmakov et al. 2016) & $\sim 22.5 \mathrm{k}$ & $\checkmark$ & 69.7 & 82.9 & 5.6 & 66.3 & 78.3 & 6.7 & 68.6 \\
\hline Flow saliency & 0 & $\checkmark$ & 70.7 & 83.2 & 6.7 & 69.7 & 82.9 & 7.9 & 48.2 \\
\hline FusionSeg (Jain et al. 2017) & $\sim 95 \mathrm{k}$ & $\checkmark$ & 71.5 & - & - & - & - & - & - \\
\hline LVO (Tokmakov et al. 2017) & $\sim 35 \mathrm{k}$ & $\checkmark$ & 75.9 & 89.1 & 0.0 & 72.1 & 83.4 & 1.3 & 26.5 \\
\hline PDB (Song et al. 2018) & $\sim 18 \mathrm{k}$ & $x$ & 77.2 & 90.1 & 0.9 & 74.5 & 84.4 & -0.2 & 29.1 \\
\hline \multicolumn{10}{|l|}{ Uses 1st frame annotation } \\
\hline Mask warping & 0 & $\checkmark$ & 32.1 & 25.5 & 31.7 & 36.3 & 23.0 & 32.8 & 8.4 \\
\hline FCP (Perazzi et al. 2015) & 0 & $\checkmark$ & 63.1 & 77.8 & 3.1 & 54.6 & 60.4 & 3.9 & 28.5 \\
\hline BVS (Maerki et al. 2016) & 0 & $x$ & 66.5 & 76.4 & 26.0 & 65.6 & 77.4 & 23.6 & 31.6 \\
\hline ObjFlow (Tsai et al. 2016) & 0 & $\checkmark$ & 71.1 & 80.0 & 22.7 & 67.9 & 78.0 & 24.0 & 22.1 \\
\hline STV (Wang and Shen 2017) & 0 & $\sqrt{ }$ & 73.6 & - & - & 72.0 & - & - & - \\
\hline VPN (Jampani et al. 2016) & $\sim 2.3 \mathrm{k}$ & $x$ & 75.0 & - & - & 72.4 & - & - & 29.5 \\
\hline OSVOS (Caelles et al. 2017) & $\sim 2.3 \mathrm{k}$ & $x$ & 79.8 & 93.6 & 14.9 & 80.6 & 92.6 & 15.0 & 37.6 \\
\hline MaskTrack (Khoreva et al. 2016) & $\sim 11 \mathrm{k}$ & $\checkmark$ & 80.3 & 93.5 & 8.9 & 75.8 & 88.2 & 9.5 & 18.3 \\
\hline PReMVOS (Luiten and Voigtlaender 2018) & $\sim 145 \mathrm{k}$ & $\checkmark$ & 84.9 & 96.1 & 8.8 & 88.6 & 94.7 & 9.8 & 19.7 \\
\hline OnAVOS (Voigtlaender and Leibe 2017b) & $\sim 120 \mathrm{k}$ & $x$ & 86.1 & 96.1 & 5.2 & 84.9 & 89.7 & 5.8 & 19.0 \\
\hline VideoGCRF (Chandra et al. 2018) & $\sim 120 \mathrm{k}$ & $x$ & 86.5 & - & - & - & - & - & - \\
\hline LucidTracker & 50 & $\checkmark$ & 86.6 & 97.3 & 5.3 & 84.8 & 93.1 & 7.5 & 15.9 \\
\hline
\end{tabular}

Numbers in italic are reported on subsets of DAVIS ${ }_{16}$, and in bold are the best numbers overall, and in bolditalic are reported on subsets of DAVIS 16 and the best numbers overall

sions: it might takes several frames for the full object mask to re-appear (rows $1-3$ in Fig. 10). This is mainly due to the convnet having learnt to follow-up the previous frame mask. Augmenting the lucid dreams with plausible occlusions might help mitigate this case. Another failure case occurs when two similar looking objects cross each other, as in row 6 in Fig. 10. Here both cues: the previous frame guidance and learnt via per-video tuning appearance, are no longer discriminative to correctly continue propagating the mask.

We also observe that the LucidTracker struggles to track the fine structures or details of the object, e.g. wheels of the bicycle or motorcycle in rows 1-2 in Fig. 10. This is the issue of the underlying choice of the convnet architecture, due to the several pooling layers the spatial resolution is lost and hence the fine details of the object are missing. This issue can be mitigated by switching to more recent semantic labelling architectures (e.g. Pohlen et al. 2017; Chen et al. 2017).

Conclusion LucidTracker shows robust performance across different videos. However, a few failure cases were observed due to the underlying convnet architecture, its training, or limited visibility of the object in the first frame.

\section{Multiple Object Segmentation Results}

We present here an empirical evaluation of LucidTracker for multiple object segmentation task: given a first frame labelled with the masks of several object instances, one aims to find the corresponding masks of objects in future frames. 
Table 10 DAVIS 16 per-attribute evaluation. LucidTracker improves across all video object segmentation challenges

\begin{tabular}{|c|c|c|c|c|c|}
\hline \multirow[t]{2}{*}{ Attribute } & \multicolumn{5}{|l|}{ Method } \\
\hline & $\begin{array}{l}\text { BVS (Maerki } \\
\text { et al. 2016) }\end{array}$ & $\begin{array}{l}\text { ObjFlow (Tsai } \\
\text { et al. 2016) }\end{array}$ & $\begin{array}{l}\text { OSVOS (Caelles } \\
\text { et al. 2017) }\end{array}$ & $\begin{array}{l}\text { MaskTrack (Khoreva } \\
\text { et al. 2016) }\end{array}$ & LucidTracker \\
\hline Appearance change & 0.46 & 0.54 & 0.81 & 0.76 & 0.84 \\
\hline Background clutter & 0.63 & 0.68 & 0.83 & 0.79 & 0.86 \\
\hline Camera-shake & 0.62 & 0.72 & 0.78 & 0.78 & 0.88 \\
\hline Deformation & 0.7 & 0.77 & 0.79 & 0.78 & 0.87 \\
\hline Dynamic background & 0.6 & 0.67 & 0.74 & 0.76 & 0.82 \\
\hline Edge ambiguity & 0.58 & 0.65 & 0.77 & 0.74 & 0.82 \\
\hline Fast-motion & 0.53 & 0.55 & 0.76 & 0.75 & 0.85 \\
\hline Heterogeneous object & 0.63 & 0.66 & 0.75 & 0.79 & 0.85 \\
\hline Interacting objects & 0.63 & 0.68 & 0.75 & 0.77 & 0.85 \\
\hline Low resolution & 0.59 & 0.58 & 0.77 & 0.77 & 0.84 \\
\hline Motion blur & 0.58 & 0.6 & 0.74 & 0.74 & 0.83 \\
\hline Occlusion & 0.68 & 0.66 & 0.77 & 0.77 & 0.84 \\
\hline Out-of-view & 0.43 & 0.53 & 0.72 & 0.71 & 0.84 \\
\hline Scale variation & 0.49 & 0.56 & 0.74 & 0.73 & 0.81 \\
\hline Shape complexity & 0.67 & 0.69 & 0.71 & 0.75 & 0.82 \\
\hline
\end{tabular}

Numbers in italic are reported on subsets of DAVIS 16 and in bold are the best numbers overall

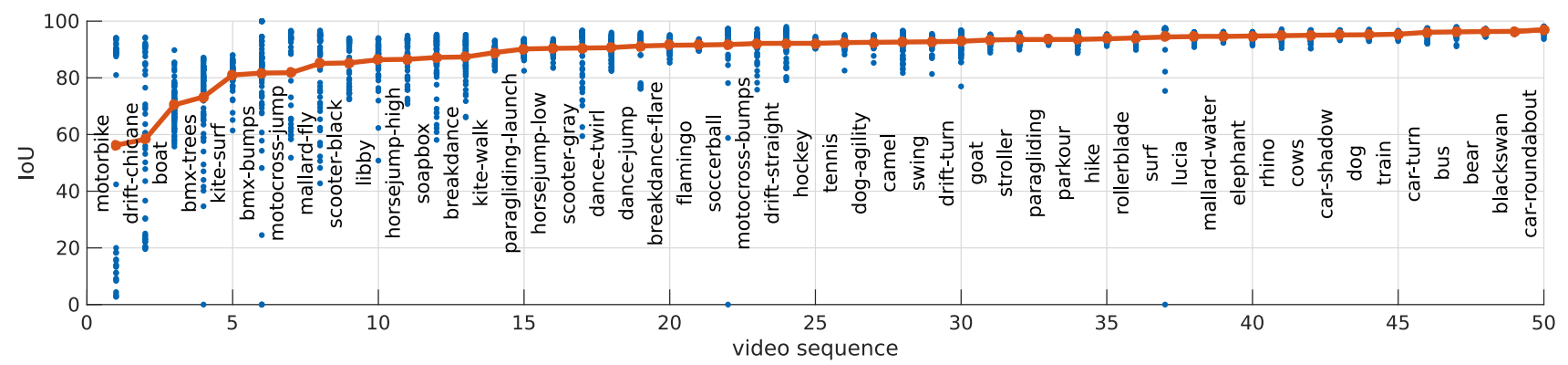

Fig. 9 Per-sequence results on DAVIS 16

\subsection{Experimental Setup}

Dataset For the multiple object segmentation task we use the 2017 DAVIS Challenge on Video Object Segmentation $^{2}$ (Pont-Tuset et al. 2017b) (DAVIS ${ }_{17}$ ). Compared to DAVIS $_{16}$ this is a larger, more challenging dataset, where the video sequences have multiple objects in the scene. Videos that have more than one visible object in DAVIS $_{16}$ have been re-annotated (the objects were divided by semantics) and the train and val sets were extended with more sequences. In addition, two other test sets (test-dev and testchallenge) were introduced. The complexity of the videos has increased with more distractors, occlusions, fast motion, smaller objects, and fine structures. Overall, DAVIS ${ }_{17}$ consists of 150 sequences, totalling 10474 annotated frames and 384 objects.

\footnotetext{
${ }^{2}$ http://davischallenge.org/challenge2017.
}

We evaluate our method on two test sets, the test-dev and test-challenge sets, each consists of 30 video sequences, on average $\sim 3$ objects per sequence, the length of the sequences is $\sim 70$ frames. For both test sets only the masks on the first frames are made public, the evaluation is done via an evaluation server. Our experiments and ablation studies are done on the test-dev set.

Evaluation Metric The accuracy of multiple object segmentation is evaluated using the region $(\mathrm{J})$ and boundary $(\mathrm{F})$ measures proposed by the organisers of the challenge. The average of $\mathrm{J}$ and $\mathrm{F}$ measures is used as overall performance score (denoted as global mean in the tables). Please refer to Pont-Tuset et al. (2017b) for more details about the evaluation protocol.

Training Details All experiments in this section are done using the single stream architecture discussed in Sects. 3.1 and 5.4.3. For training the models we use SGD with minibatches of 10 images and a fixed learning policy with initial 

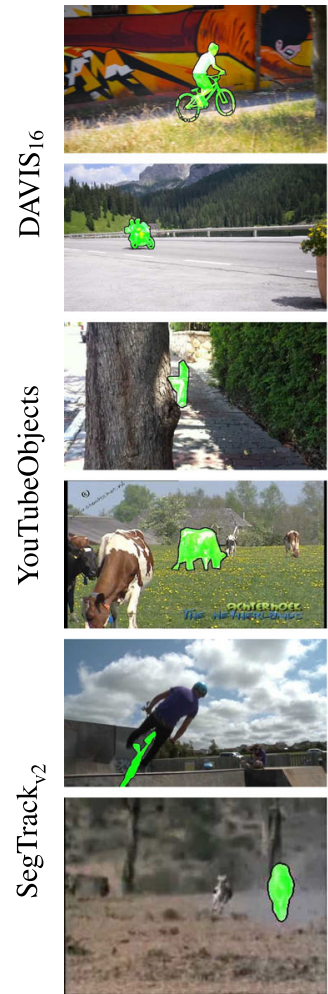

1st frame, GT segment
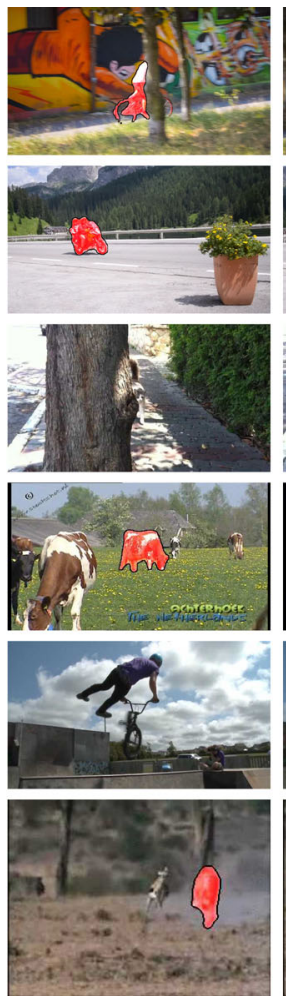

$20 \%$
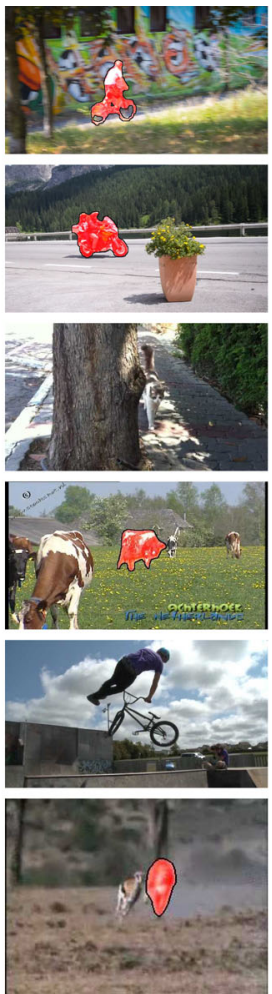

$40 \%$
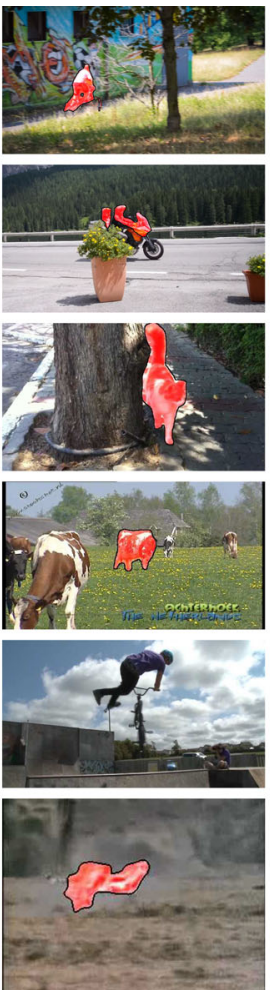

$60 \%$
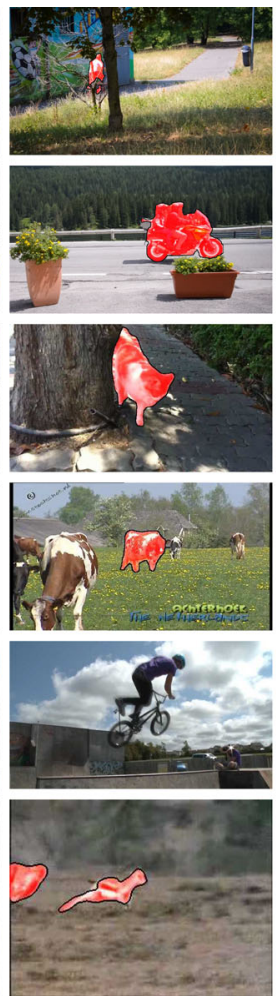

$80 \%$
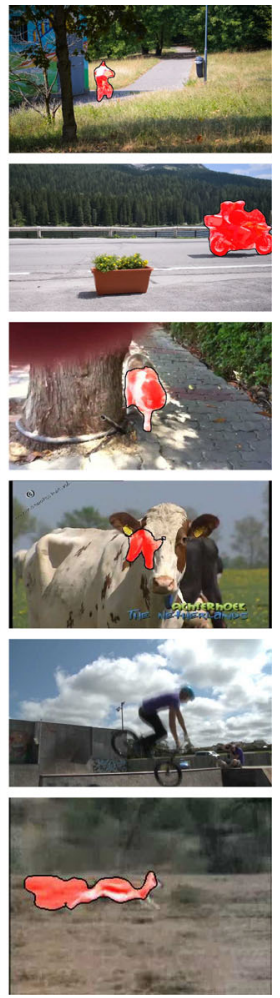

$100 \%$

Fig. 10 Failure cases. Frames sampled along the video duration (e.g. 50\%: video middle point). For each dataset we show 2 out of 5 worst results (based on mIoU over the video)

learning rate of $10^{-3}$. The momentum and weight decay are set to 0.9 and $5 \times 10^{-4}$, respectively. All models are initialized with weights trained for image classification on ImageNet (Simonyan and Zisserman 2015). We then train per-video for $40 \mathrm{k}$ iterations.

\subsection{Key Results}

Tables 11 and 12 presents the results of the 2017 DAVIS Challenge on test-dev and test-challenge sets (Pont-Tuset et al. 2017a).

Our main results for the multi-object segmentation challenge are obtained via an ensemble of four different models $\left(f_{\mathcal{I}}, f_{\mathcal{I}+\mathcal{F}}, f_{\mathcal{I}+\mathcal{S}}, f_{\mathcal{I}+\mathcal{F}+\mathcal{S}}\right)$, see Sect. 3.1.

The proposed system, LucidTracker, provides the best segmentation quality on the test-dev set and shows competitive performance on the test-challenge set, holding the second place in the competition. The full system is trained using the standard ImageNet pre-training initialization, Pascal VOC12 semantic annotations for the $\mathcal{S}_{t}$ input $(\sim 10 \mathrm{k}$ annotated images), and one annotated frame per test video, 30 frames total on each test set. As discussed in Sect. 6.3, even without $\mathcal{S}_{t}$ LucidTracker obtains competitive results ( $<1 \%$ point difference, see Table 13 for details).
The top entry lixx (Li et al. 2017) uses a deeper convnet model (ImageNet pre-trained ResNet), a similar segmentation architecture, trains it over external segmentation data (using $\sim 120 \mathrm{k}$ pixel-level annotated images from MS-COCO and Pascal VOC for pre-training, and akin to Caelles et al. (2017) fine-tuning on the DAVIS ${ }_{17}$ train and val sets, $\sim 10 \mathrm{k}$ annotated frames), and extends it with a box-level object detector (trained over MS-COCO and Pascal VOC, $\sim 500 \mathrm{k}$ bounding boxes) and a box-level object reidentification model trained over $\sim 60 \mathrm{k}$ box annotations (on both images and videos). We argue that our system reaches comparable results with a significantly lower amount of training data.

Figure 11 provides qualitative results of LucidTracker on the test-dev set. The video results include successful handling of multiple objects, full and partial occlusions, distractors, small objects, and out-of-view scenarios.

Conclusion We show that top results for multiple object segmentation can be achieved via our approach that focuses on exploiting as much as possible the available annotation on the first video frame, rather than relying heavily on large external training data. 
Table 11 Comparison of video object segmentation results on DAVIS ${ }_{17}$, test-dev set. Our LucidTracker shows top performance

\begin{tabular}{|c|c|c|c|c|c|c|c|c|}
\hline \multirow[t]{3}{*}{ Method } & \multicolumn{8}{|c|}{ DAVIS $_{17}$, test-dev set } \\
\hline & \multirow[t]{2}{*}{ Rank } & \multirow[t]{2}{*}{ Global mean $\uparrow$} & \multicolumn{3}{|c|}{ Region, $J$} & \multicolumn{3}{|c|}{ Boundary, $F$} \\
\hline & & & Mean $\uparrow$ & Recall $\uparrow$ & Decay $\downarrow$ & Mean $\uparrow$ & Recall $\uparrow$ & Decay $\downarrow$ \\
\hline sidc & 10 & 45.8 & 43.9 & 51.5 & 34.3 & 47.8 & 53.6 & 36.9 \\
\hline YXLKJ & 9 & 49.6 & 46.1 & 49.1 & 22.7 & 53.0 & 56.5 & 22.3 \\
\hline haamooon (Shaban et al. 2017) & 8 & 51.3 & 48.8 & 56.9 & 12.2 & 53.8 & 61.3 & 11.8 \\
\hline Fromandtozh (Zhao 2017) & 7 & 55.2 & 52.4 & 58.4 & 18.1 & 57.9 & 66.1 & 20.0 \\
\hline ilanv (Sharir et al. 2017) & 6 & 55.8 & 51.9 & 55.7 & 17.6 & 59.8 & 65.8 & 18.9 \\
\hline voigtlaender (Voigtlaender and Leibe 2017a) & 5 & 56.5 & 53.4 & 57.8 & 19.9 & 59.6 & 65.4 & 19.0 \\
\hline lalalafine 123 & 4 & 57.4 & 54.5 & 61.3 & 24.4 & 60.2 & 68.8 & 24.6 \\
\hline wangzhe & 3 & 57.7 & 55.6 & 63.2 & 31.7 & 59.8 & 66.7 & 37.1 \\
\hline lixx (Li et al. 2017) & 2 & 66.1 & 64.4 & 73.5 & 24.5 & 67.8 & 75.6 & 27.1 \\
\hline LucidTracker & 1 & 66.6 & 63.4 & 73.9 & 19.5 & 69.9 & 80.1 & 19.4 \\
\hline
\end{tabular}

Bold are the best numbers overall

Table 12 Comparison of video object segmentation results on DAVIS ${ }_{17}$, test-challenge set. Our LucidTracker shows competitive performance, holding the second place in the competition

\begin{tabular}{|c|c|c|c|c|c|c|c|c|}
\hline \multirow[t]{3}{*}{ Method } & \multicolumn{8}{|c|}{ DAVIS $_{17}$, test-challenge set } \\
\hline & \multirow[t]{2}{*}{ Rank } & \multirow[t]{2}{*}{ Global mean $\uparrow$} & \multicolumn{3}{|c|}{$\underline{\text { Region, } J}$} & \multicolumn{3}{|c|}{ Boundary, $F$} \\
\hline & & & Mean $\uparrow$ & Recall $\uparrow$ & Decay $\downarrow$ & Mean $\uparrow$ & Recall $\uparrow$ & Decay $\downarrow$ \\
\hline zwrq0 & 10 & 53.6 & 50.5 & 54.9 & 28.0 & 56.7 & 63.5 & 30.4 \\
\hline Fromandtozh (Zhao 2017) & 9 & 53.9 & 50.7 & 54.9 & 32.5 & 57.1 & 63.2 & 33.7 \\
\hline wasidennis & 8 & 54.8 & 51.6 & 56.3 & 26.8 & 57.9 & 64.8 & 28.8 \\
\hline YXLKJ & 7 & 55.8 & 53.8 & 60.1 & 37.7 & 57.8 & 62.1 & 42.9 \\
\hline cjc (Cheng et al. 2017) & 6 & 56.9 & 53.6 & 59.5 & 25.3 & 60.2 & 67.9 & 27.6 \\
\hline lalalafine 123 & 6 & 56.9 & 54.8 & 60.7 & 34.4 & 59.1 & 66.7 & 36.1 \\
\hline voigtlaender (Voigtlaender and Leibe 2017a) & 5 & 57.7 & 54.8 & 60.8 & 31.0 & 60.5 & 67.2 & 34.7 \\
\hline haamooon (Shaban et al. 2017) & 4 & 61.5 & 59.8 & 71.0 & 21.9 & 63.2 & 74.6 & 23.7 \\
\hline vantam299 (Le et al. 2017) & 3 & 63.8 & 61.5 & 68.6 & 17.1 & 66.2 & 79.0 & 17.6 \\
\hline LucidTracker & 2 & 67.8 & 65.1 & 72.5 & 27.7 & 70.6 & 79.8 & 30.2 \\
\hline lixx (Li et al. 2017) & 1 & 69.9 & 67.9 & 74.6 & 22.5 & 71.9 & 79.1 & 24.1 \\
\hline
\end{tabular}

Bold are the best numbers overall

\subsection{Ablation Study}

Table 13 explores in more details how the different ingredients contribute to our results.

We see that adding extra information (channels) to the system, either optical flow magnitude or semantic segmentation, or both, does provide $1-2 \%$ point improvement. The results show that leveraging semantic priors and motion information provides a complementary signal to RGB image and both ingredients contribute to the segmentation results.

Combining in ensemble four different models $\left(f_{\mathcal{I}+\mathcal{F}+\mathcal{S}}+\right.$ $\left.f_{\mathcal{I}+\mathcal{F}}+f_{\mathcal{I}+\mathcal{S}}+f_{\mathcal{I}}\right)$ allows to enhance the results even further, bringing $2.7 \%$ point gain $(62.0$ vs. 64.7 global mean). Excluding the models which use semantic information $\left(f_{\mathcal{I}+\mathcal{F}+\mathcal{S}}\right.$ and $\left.f_{\mathcal{I}+\mathcal{S}}\right)$ from the ensemble results only in a minor drop in the performance (64.2 vs. 64.7 global mean). This shows that the competitive results can be achieved even with the system trained only with one pixel-level mask annotation per video, without employing extra annotations from Pascal VOC12.

Our lucid dreams enable automatic CRF-tuning (see Sect. 5.3.3) which allows to further improve the results $(64.7 \rightarrow 65.2$ global mean). Employing the proposed temporal coherency step (see Sect. 3.1) during inference brings an additional performance gain $(65.2 \rightarrow 66.6$ global mean).

Conclusion The results show that both flow and semantic priors provide a complementary signal to RGB image only. Despite its simplicity our ensemble strategy provides addi- 


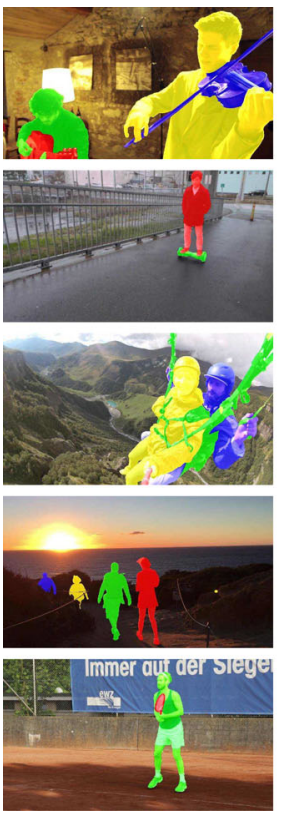

1st frame, GT segment
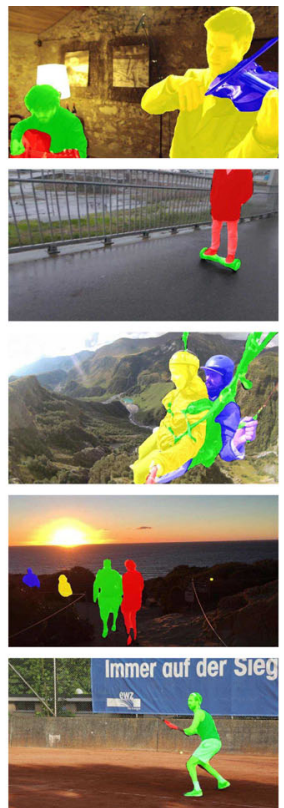

$20 \%$
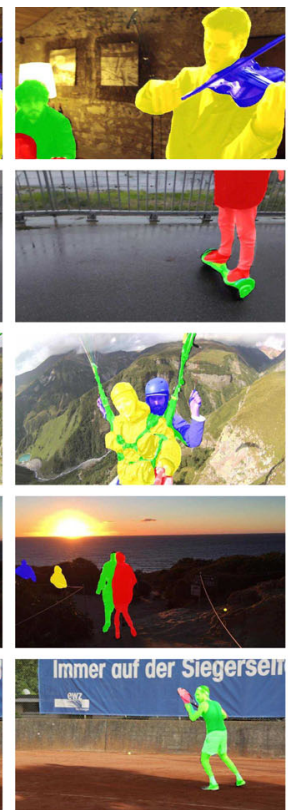

$40 \%$
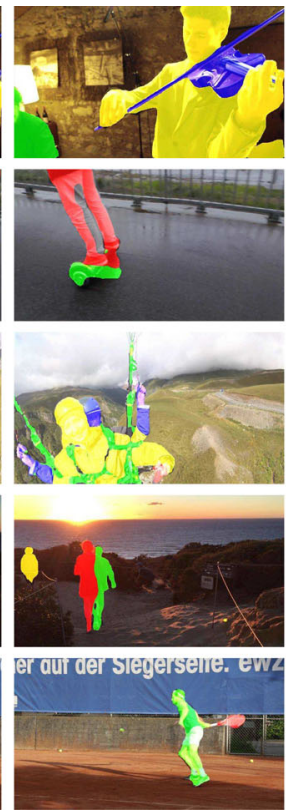

$60 \%$
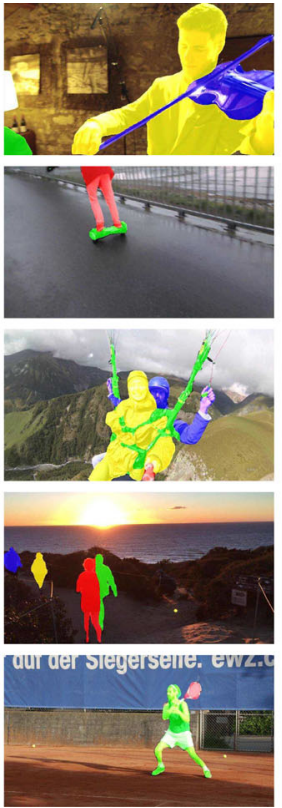

$80 \%$

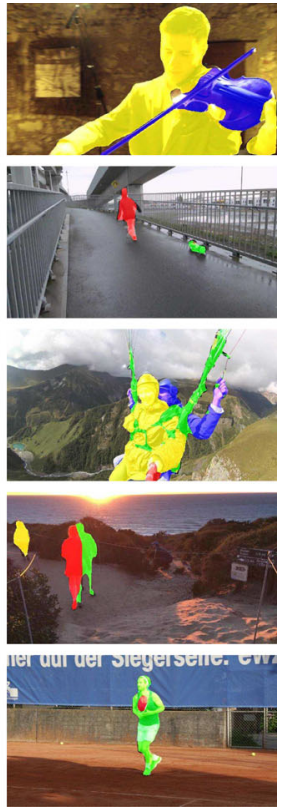

$100 \%$

Fig. 11 LucidTracker qualitative results on DAVIS ${ }_{17}$, test-dev set. Frames sampled along the video duration (e.g. 50\%: video middle point). The videos are chosen with the highest mIoU measure

Table 13 Ablation study of different ingredients. DAVIS ${ }_{17}$, test-dev and test challenge sets

\begin{tabular}{|c|c|c|c|c|c|c|c|c|c|c|c|c|}
\hline \multirow[t]{3}{*}{ Variant } & \multirow[t]{3}{*}{$\mathcal{I}$} & \multirow[t]{3}{*}{$\mathcal{F}$} & \multirow[t]{3}{*}{$\mathcal{S}$} & \multirow[t]{3}{*}{ Ensemble } & \multirow[t]{3}{*}{ CRF tuning } & \multirow[t]{3}{*}{ Temp. coherency } & \multicolumn{6}{|c|}{ DAVIS $_{17}$} \\
\hline & & & & & & & \multicolumn{2}{|c|}{ Test-dev } & \multicolumn{4}{|c|}{ Test-challenge } \\
\hline & & & & & & & Global mean & $\mathrm{mIoU}$ & $\mathrm{mF}$ & Global mean & $\mathrm{mIoU}$ & $\mathrm{mF}$ \\
\hline \multirow[t]{5}{*}{ LucidTracker (ensemble) } & $\checkmark$ & $\checkmark$ & $\checkmark$ & $\checkmark$ & $\checkmark$ & $\checkmark$ & 66.6 & 63.4 & 69.9 & 67.8 & 65.1 & 70.6 \\
\hline & $\checkmark$ & $\checkmark$ & $\sqrt{ }$ & $\sqrt{ }$ & $\checkmark$ & $x$ & 65.2 & 61.5 & 69.0 & 67.0 & 64.3 & 69.7 \\
\hline & $\checkmark$ & $\checkmark$ & $\sqrt{ }$ & $\checkmark$ & $x$ & $x$ & 64.7 & 60.5 & 68.9 & 66.5 & 63.2 & 69.8 \\
\hline & $\checkmark$ & $\checkmark$ & $x$ & $\checkmark$ & $\checkmark$ & $x$ & 64.9 & 61.3 & 68.4 & - & - & - \\
\hline & $\checkmark$ & $\checkmark$ & $x$ & $\checkmark$ & $x$ & $x$ & 64.2 & 60.1 & 68.3 & - & - & - \\
\hline LucidTracker & $\checkmark$ & $\checkmark$ & $\sqrt{ }$ & $x$ & $\checkmark$ & $x$ & 62.9 & 59.1 & 66.6 & - & - & - \\
\hline $\mathcal{I}+\mathcal{F}+\mathcal{S}$ & $\checkmark$ & $\checkmark$ & $\sqrt{ }$ & $x$ & $x$ & $x$ & 62.0 & 57.7 & 62.2 & 64.0 & 60.7 & 67.3 \\
\hline $\mathcal{I}+\mathcal{F}$ & $\checkmark$ & $\checkmark$ & $x$ & $x$ & $x$ & $x$ & 61.3 & 56.8 & 65.8 & - & - & - \\
\hline $\mathcal{I}+\mathcal{S}$ & $\checkmark$ & $x$ & $\sqrt{ }$ & $x$ & $x$ & $x$ & 61.1 & 56.9 & 65.3 & - & - & - \\
\hline $\mathcal{I}$ & $\checkmark$ & $x$ & $x$ & $x$ & $x$ & $x$ & 59.8 & 63.1 & 63.9 & - & - & - \\
\hline
\end{tabular}

Bold are the best numbers overall

tional gain and leads to competitive results. Notice that even without the semantic segmentation signal $\mathcal{S}_{t}$ our ensemble result is competitive.

\subsection{Error Analysis}

We present the per-sequence results of LucidTracker on DAVIS $_{17}$ in Figure 12 (per frame results not available from evaluation server). We observe that this dataset is significantly more challenging than DAVIS $_{16}$ (compare to Figure
9), with only $1 / 3$ of the test videos above $80 \mathrm{mIoU}$. This shows that multiple object segmentation is a much more challenging task than segmenting a single object.

The failure cases discussed in Sect. 5.5 still apply to the multiple objects case. Additionally, on DAVIS 17 we observe a clear failure case when segmenting similar looking object instances, where the object appearance is not discriminative to correctly track the object, resulting in label switches or bleeding of the label to other look-alike objects. Figure 13 illustrates this case. This issue could be mitigated by using 


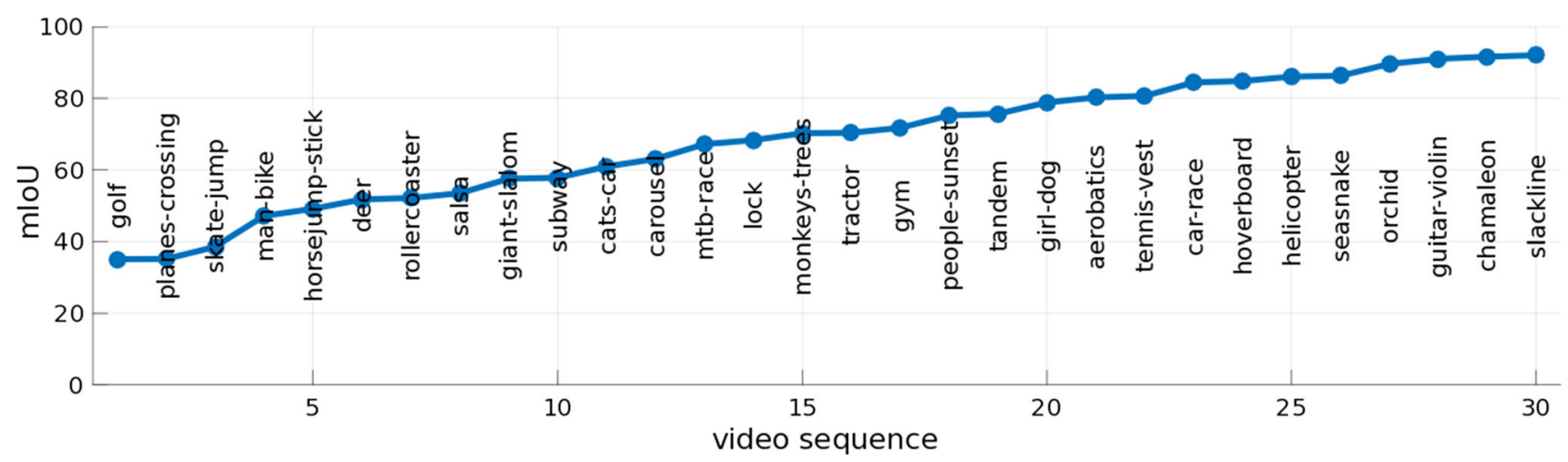

Fig. 12 Per-sequence results on DAVIS ${ }_{17}$, test-dev set
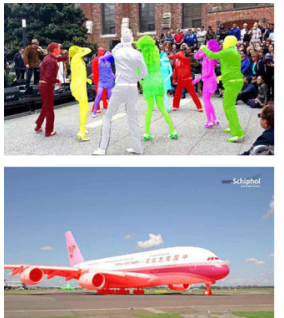

1st frame, GT segment
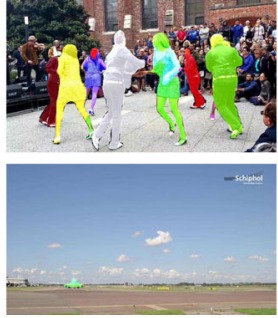

$20 \%$
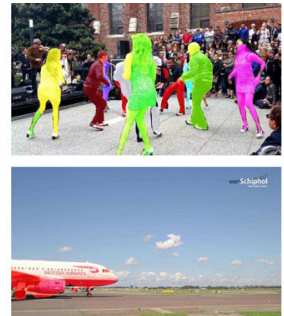

$40 \%$
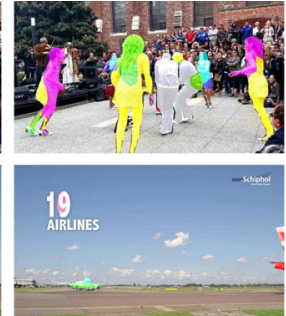

$60 \%$
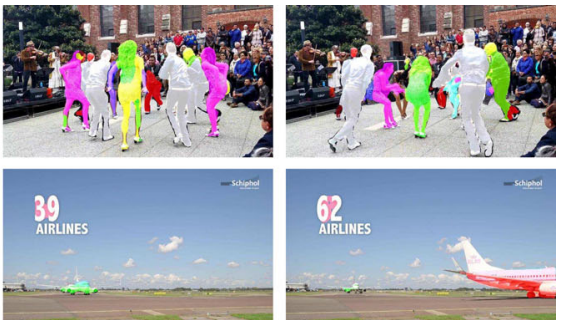

$80 \%$

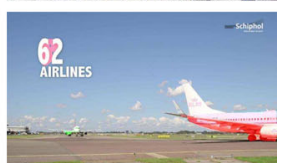

$100 \%$

Fig. 13 LucidTracker failure cases on DAVIS ${ }_{17}$, test-dev set. Frames sampled along the video duration (e.g. 50\%: video middle point). We show 2 results mIoU over the video below 50

object level instance identification modules, like ( $\mathrm{Li}$ et al. 2017), or by changing the training loss of the model to more severely penalize identity switches.

Conclusion In the multiple object case the LucidTracker results remain robust across different videos. The overall results being lower than for the single object segmentation case, there is more room for future improvement in the multiple object pixel-level segmentation task.

\section{Conclusion}

We have described a new convnet-based approach for pixellevel object segmentation in videos. In contrast to previous work, we show that top results for single and multiple object segmentation can be achieved without requiring external training datasets (neither annotated images nor videos). Even more, our experiments indicate that it is not always beneficial to use additional training data, synthesizing training samples close to the test domain is more effective than adding more training samples from related domains.

Our extensive analysis decomposed the ingredients that contribute to our improved results, indicating that our new training strategy and the way we leverage additional cues such as semantic and motion priors are key.
Showing that training a convnet for video object segmentation can be done with only few $(\sim 100)$ training samples changes the mindset regarding how much general knowledge about objects is required to approach this problem (Khoreva et al. 2016; Jain et al. 2017), and more broadly how much training data is required to train large convnets depending on the task at hand.

We hope these new results will fuel the ongoing evolution of convnet techniques for single and multiple object segmentation in videos.

Acknowledgements Open access funding provided by Max Planck Society. Eddy Ilg and Thomas Brox acknowledge funding by the DFG Grant BR 3815/7-1.

Open Access This article is distributed under the terms of the Creative Commons Attribution 4.0 International License (http://creativecomm ons.org/licenses/by/4.0/), which permits unrestricted use, distribution, and reproduction in any medium, provided you give appropriate credit to the original author(s) and the source, provide a link to the Creative Commons license, and indicate if changes were made.

\section{References}

Aytekin, Ç., Ozan, E. C., Kiranyaz, S., \& Gabbouj, M. (2015). Visual saliency by extended quantum cuts. In ICIP.

Bansal, A., Chen, X., Russell, B., Gupta, A., \& Ramanan, D. (2017). Pixelnet: Representation of the pixels, by the pixels, and for the pixels. arXiv:1702.06506. 
Bertinetto, L., Valmadre, J., Henriques, J. F., Vedaldi, A., \& Torr, P. H. (2016). Fully-convolutional siamese networks for object tracking. arXiv: 1606.09549

Bookstein, F. (1989). Principal warps: Thin-plate splines and the decomposition of deformations. IEEE Transactions on Pattern Analysis \& Machine Intelligence, 6, 567-585.

Breitenstein, M. D., Reichlin, F., Leibe, B., Koller-Meier, E., \& Van Gool, L. (2009). Robust tracking-by-detection using a detector confidence particle filter. In ICCV.

Caelles, S., Maninis, K.-K., Pont-Tuset, J., Leal-Taixe, L., Cremers, D., \& Gool, L. V. (2017). One-shot video object segmentation. In CVPR.

Chandra, S., Couprie, C., \& Kokkinos, I. (2018). Deep spatio-temporal random fields for efficient video segmentation. In CVPR.

Chang, J., Wei, D., \& Fisher, J. W. (2013). A video representation using temporal superpixels. In $C V P R$.

Chen, L., Papandreou, G., Schroff, F., \& Adam, H. (2017). Rethinking atrous convolution for semantic image segmentation. arXiv: 1706.05587.

Chen, L.-C., Papandreou, G., Kokkinos, I., Murphy, K., \& Yuille, A. L. (2016). Deeplab: Semantic image segmentation with deep convolutional nets, atrous convolution, and fully connected CRFs. arXiv:1606.00915.

Chen, W., Wang, H., Li, Y., Su, H., Wang, Z., Tu, C., et al. (2016). Synthesizing training images for boosting human 3D pose estimation. In $3 D$ Vision $(3 D V)$.

Cheng, J., Liu, S., Tsai, Y.-H., Hung, W.-C., Gupta, S., Gu, J., et al. (2017). Learning to segment instances in videos with spatial propagation network. In CVPR workshops.

Criminisi, A., Perez, P., \& Toyama, K. (2004). Region filling and object removal by exemplar-based image inpainting. IEEE Transactions on Image Processing, 13(9), 1200-1212.

Danelljan, M., Hager, G., Shahbaz Khan, F., \& Felsberg, M. (2015). Convolutional features for correlation filter based visual tracking. In ICCV workshop.

Dosovitskiy, A., Fischer, P., Ilg, E., Häusser, P., Hazirbas, C., Golkov, V., et al. (2015). Flownet: Learning optical flow with convolutional networks. In ICCV.

Everingham, M., Eslami, S. M. A., Van Gool, L., Williams, C. K. I., Winn, J., \& Zisserman, A. (2015). The pascal visual object classes challenge: A retrospective. International Journal of Computer Vision, 111(1), 98-136.

Faktor, A., \& Irani, M. (2014). Video segmentation by non-local consensus voting. In $B M V C$.

Georgakis, G., Mousavian, A., Berg, A. C., \& Kosecka, J. (2017). Synthesizing training data for object detection in indoor scenes. arXiv: 1702.07836.

Glorot, X., \& Bengio, Y. (2010). Understanding the difficulty of training deep feedforward neural networks. In AISTATS.

Grundmann, M., Kwatra, V., Han, M., \& Essa, I. (2010). Efficient hierarchical graph-based video segmentation. In $C V P R$.

Gupta, A., Vedaldi, A., \& Zisserman, A. (2016). Synthetic data for text localisation in natural images. In $C V P R$.

Held, D., Thrun, S., \& Savarese, S. (2016). Learning to track at $100 \mathrm{fps}$ with deep regression networks. In $E C C V$.

Henriques, J. F., Caseiro, R., Martins, P., \& Batista, J. (2012). Exploiting the circulant structure of tracking-by-detection with kernels. In ECCV.

Ilg, E., Mayer, N., Saikia, T., Keuper, M., Dosovitskiy, A., \& Brox, T. (2017). Flownet 2.0: Evolution of optical flow estimation with deep networks. In $C V P R$.

Luiten, B. L. J., \& Voigtlaender, P. (2018). PReMVOS: Proposalgeneration, refinement and merging for video object segmentation. In $A C C V$.

Jain, S. D., \& Grauman, K. (2014). Supervoxel-consistent foreground propagation in video. In $E C C V$.
Jain, S. D., \& Grauman, K. (2016). Click carving: Segmenting objects in video with point clicks. In HCOMP.

Jain, S. D., Xiong, B., \& Grauman, K. (2017). Fusionseg: Learning to combine motion and appearance for fully automatic segmention of generic objects in videos. arXiv: 1701.05384.

Jampani, V., Gadde, R., \& Gehler, P. V. (2016). Video propagation networks. arXiv: 1612.05478 .

Khoreva, A., Benenson, R., Ilg, E., Brox, T., \& Schiele, B. (2017). Lucid data dreaming for object tracking. In CVPR workshops.

Khoreva, A., Perazzi, F., Benenson, R., Schiele, B., \& Sorkine-Hornung, A. (2016). Learning video object segmentation from static images. arXiv:1612.02646.

Krähenbühl, P., \& Koltun, V. (2011). Efficient inference in fully connected CRFs with Gaussian edge potentials. In NIPS.

Kristan, M., Matas, J., Leonardis, A., Felsberg, M., Cehovin, L., Fernandez, G., et al. (2014). The visual object tracking VOT2014 challenge results. In ECCV workshop.

Kristan, M., Matas, J., Leonardis, A., Felsberg, M., Cehovin, L., Fernandez, G., et al. (2015). The visual object tracking VOT2015 challenge results. In ICCV workshop.

Kristan, M., Matas, J., Leonardis, A., Felsberg, M., Cehovin, L., Fernandez, G., et al. (2016). The visual object tracking VOT2016 challenge results. In ECCV workshop.

Le, T. N., Nguyen, K. T., Nguyen-Phan, M. H., Ton, T. V., Nguyen, T. A., Trinh, X. S., et al. (2017). Instance re-identification flow for video object segmentation. In CVPR workshops.

Li, F., Kim, T., Humayun, A., Tsai, D., \& Rehg, J. M. (2013). Video segmentation by tracking many figure-ground segments. In ICCV.

Li, X., Qi, Y., Wang, Z., Chen, K., Liu, Z., Shi, J., et al. (2017). Video object segmentation with re-identification. In CVPR workshops.

Lin, G., Milan, A., Shen, C., \& Reid, I. D. (2016). Refinenet: Multi-path refinement networks for high-resolution semantic segmentation. arXiv: 1611.06612.

Ma, C., Huang, J.-B., Yang, X., \& Yang, M.-H. (2015). Hierarchical convolutional features for visual tracking. In ICCV.

Maerki, N., Perazzi, F., Wang, O., \& Sorkine-Hornung, A. (2016). Bilateral space video segmentation. In $C V P R$.

Nagaraja, N., Schmidt, F., \& Brox, T. (2015). Video segmentation with just a few strokes. In $I C C V$.

Nam, H., Baek, M., \& Han, B. (2016). Modeling and propagating CNNS in a tree structure for visual tracking. arXiv:1608.07242.

Nam, H., \& Han, B. (2016). Learning multi-domain convolutional neural networks for visual tracking. In $C V P R$.

Mayer, N., Ilg, E., Häusser, P., Fischer, P., Cremers, D., Dosovitskiy, A., et al. (2016). A large dataset to train convolutional networks for disparity, optical flow, and scene flow estimation. In $C V P R$.

Papazoglou, A., \& Ferrari, V. (2013). Fast object segmentation in unconstrained video. In $I C C V$.

Park, D., \& Ramanan, D. (2015). Articulated pose estimation with tiny synthetic videos. In CVPR workshop.

Pathak, D., Girshick, R., Dollár, P., Darrell, T., \& Hariharan, B. (2016). Learning features by watching objects move. arXiv:1612.06370.

Perazzi, F., Pont-Tuset, J., McWilliams, B., Gool, L. V., Gross, M., \& Sorkine-Hornung, A. (2016). A benchmark dataset and evaluation methodology for video object segmentation. In CVPR.

Perazzi, F., Wang, O., Gross, M., \& Sorkine-Hornung, A. (2015). Fully connected object proposals for video segmentation. In ICCV.

Pishchulin, L., Jain, A., Andriluka, M., Thormählen, T., \& Schiele, B. (2012). Articulated people detection and pose estimation: Reshaping the future. In CVPR.

Pohlen, T., Hermans, A., Mathias, M., \& Leibe, B. (2017). Fullresolution residual networks for semantic segmentation in street scenes. In $C V P R$.

Pont-Tuset, J., Perazzi, F., Caelles, S., Arbeláez, P., Sorkine-Hornung, A., \& Van Gool, L. (2017a). Davis challenge on video object segmentation. http://davischallenge.org/challenge2017. 
Pont-Tuset, J., Perazzi, F., Caelles, S., Arbeláez, P., Sorkine-Hornung, A., \& Van Gool, L. (2017b). The 2017 Davis challenge on video object segmentation. arXiv:1704.00675.

Prest, A., Leistner, C., Civera, J., Schmid, C., \& Ferrari, V. (2012). Learning object class detectors from weakly annotated video. In CVPR.

Revaud, J., Weinzaepfel, P., Harchaoui, Z., \& Schmid, C. (2015). Epicflow: Edge-preserving interpolation of correspondences for optical flow. In $C V P R$.

Richter, S. R., Vineet, V., Roth, S., \& Koltun, V. (2016). Playing for data: Ground truth from computer games. In ECCV.

Rother, C., Kolmogorov, V., \& Blake, A. (2004). Grabcut: Interactive foreground extraction using iterated graph cuts. In SIGGRAPH.

Shaban, A., Firl, A., Humayun, A., Yuan, J., Wang, X., Lei, P., et al. (2017). Multiple-instance video segmentation with sequencespecific object proposals. In CVPR workshops.

Sharir, G., Smolyansky, E., \& Friedman, I. (2017). Video object segmentation using tracked object proposals. In CVPR workshops.

Simonyan, K., \& Zisserman, A. (2015). Very deep convolutional networks for large-scale image recognition. In ICLR.

Song, H., Wang, W., Zhao, S., Shen, J., \& Lam, K.-M. (2018). Pyramid dilated deeper ConvLSTM for video salient object detection. In ECCV.

Spina, T. V., \& Falcão, A. X. (2016). Fomtrace: Interactive video segmentation by image graphs and fuzzy object models. arXiv: 1606.03369 .

Sun, J., Jia, J., Tang, C.-K., \& Shum, H.-Y. (2004). Poisson matting. In SIGGRAPH

Tang, K., Ramanathan, V., Fei-fei, L., \& Koller, D. (2012). Shifting weights: Adapting object detectors from image to video. In NIPS.

Tang, M., Marin, D., Ben Ayed, I., \& Boykov, Y. (2016). Normalized cut meets MRF. In $E C C V$.

Tang, S., Andriluka, M., Milan, A., Schindler, K., Roth, S., \& Schiele, B. (2013). Learning people detectors for tracking in crowded scenes. In $I C C V$.

Tao, R., Gavves, E., \& Smeulders, A. W. (2016). Siamese instance search for tracking. arXiv:1605.05863.

Tokmakov, P., Alahari, K., \& Schmid, C. (2016). Learning motion patterns in videos. arXiv:1612.07217.
Tokmakov, P., Alahari, K., \& Schmid, C. (2017). Learning video object segmentation with visual memory. In ICCV.

Tsai, Y.-H., Yang, M.-H., \& Black, M. J. (2016). Video segmentation via object flow. In $C V P R$.

Varol, G., Romero, J., Martin, X., Mahmood, N., Black, M. J., Laptev, I., et al. (2017). Learning from synthetic humans. arXiv:1701.01370.

Voigtlaender, P., \& Leibe, B. (2017a). Online adaptation of convolutional neural networks for the 2017 Davis challenge on video object segmentation. In CVPR workshops.

Voigtlaender, P., \& Leibe, B. (2017b). Online adaptation of convolutional neural networks for video object segmentation. In $B M V C$.

Vojir, T., \& Matas, J. (2017). Pixel-wise object segmentations for the VOT 2016 dataset. Research report.

Wang, L., Ouyang, W., Wang, X., \& Lu, H. (2015). Visual tracking with fully convolutional networks. In ICCV.

Wang, T., Han, B., \& Collomosse, J. (2014). Touchcut: Fast image and video segmentation using single-touch interaction. In CVIU.

Wang, W., \& Shen, J. (2017). Super-trajectory for video segmentation. arXiv: 1702.08634.

Wang, X., \& Gupta, A. (2015). Unsupervised learning of visual representations using videos. In ICCV.

Wu, Z., Shen, C., \& van den Hengel, A. (2016). Wider or deeper: Revisiting the ResNet model for visual recognition. arXiv:1611.10080.

Xiao, F., \& Lee, Y. J. (2016). Track and segment: An iterative unsupervised approach for video object proposals. In CVPR.

Yu, J. J., Harley, A. W., \& Derpanis, K. G. (2016). Back to basics: Unsupervised learning of optical flow via brightness constancy and motion smoothness. arXiv:1608.05842.

Zhao, H. (2017). Some promising ideas about multi-instance video segmentation. In CVPR workshops.

Zhao, H., Shi, J., Qi, X., Wang, X., \& Jia, J. (2017). Pyramid scene parsing network. In $C V P R$.

Zhu, Y., Lan, Z., Newsam, S., \& Hauptmann, A. G. (2017). Guided optical flow learning. arXiv:1702.02295.

Publisher's Note Springer Nature remains neutral with regard to jurisdictional claims in published maps and institutional affiliations. 